\title{
Characterisation of Aerotolerant Forms of a Robust Chicken Colonizing Campylobacter coli
}

\author{
Peter M. O'Kane and lan F. Connerton* \\ Division of Food Sciences, School of Biosciences, University of Nottingham, Sutton Bonington, UK
}

Campylobacter contaminated poultry meat is a major source of human foodborne illness. Campylobacter coli strain OR12 is a robust colonizer of chickens that was previously shown to outcompete and displace other Campylobacter strains from the chicken's gastrointestinal tract. This strain is capable of aerobic growth on blood agar. Serial aerobic passage increased this aerotolerance as assessed by quantitative assays for growth and survival on solid media. Aerotolerance was also associated with increased peroxide stress resistance. Aerobic passage did not alter cellular morphology or motility or hinder the microaerobic growth rate. Colonization of broiler chickens by aerotolerant $C$. coli OR12 was significantly lower than the wild-type strain at 3 days after challenge but not by 7 days, suggesting adaptation had occurred. Bacteria recovered from chickens had retained their aerotolerance, indicating this trait is stable. Whole genome sequencing enabled comparison with the wild-type sequence. Twenty-three point mutations were present, none of which were in genes known to affect oxidative stress resistance. Insertions or deletions caused frame shifts in several genes including, phosphoglycerate kinase and the $b$ subunit of pyruvate carboxylase that suggest modification of central and carbohydrate metabolism in response to aerobic growth. Other genes affected include those encoding putative carbonic anhydrase, motility accessory factor, filamentous haemagglutinin, and aminoacyl dipeptidase proteins. Aerotolerance has the potential to affect environmental success and survival. Increased environmental survival outside of the host intestinal tract may allow opportunities for transmission between hosts. Resistance to oxidative stress may equate to increased virulence by virtue of reduced susceptibility to oxidative free radicals produced by host immune responses. Finally, resistance to ambient atmospheric oxygen may allow increased survival on chicken skin, and therefore constitutes an increased risk to public health.

Keywords: Campylobacter coli, aerotolerant Campylobacter, chicken intestinal colonization, oxidative stress, Campylobacter survival, food safety

Received: 21 January 2017 Accepted: 13 March 2017 Published: 27 March 2017

Citation:

O'Kane PM and Connerton IF (2017)

Characterisation of Aerotolerant Forms of a Robust Chicken Colonizing Campylobacter coli.

Front. Microbiol. 8:513. doi: 10.3389/fmicb.2017.00513

\section{INTRODUCTION}

Campylobacter spp. have been the most commonly reported gastrointestinal pathogens in the EU since 2005, with 229,213 confirmed cases in 2015, representing 65.5 per 100,000 of population (EFSA, 2016). England and Wales reported 65,032 laboratory confirmed cases of campylobacteriosis in 2012 (PHE, 2013). This is a significant underestimate due to underdiagnosis and under-reporting. A recent study estimated that in 2009 there were 280,400 cases 
of campylobacteriosis in the UK, accounting for 38,860 general practitioner consultations and 562 hospital admissions (O'Brien et al., 2016). The economic costs associated with Campylobacter infections over the same period was estimated to be $£ 50$ million (Tam and O'Brien, 2016). The majority of these cases are associated with Campylobacter jejuni infection but it is estimated that C. coli represents $7 \%$ of the cases in the UK (Gillespie et al., 2002).

The primary feature of disease is diarrhea, which can be accompanied by abdominal pain, fever, dysentery, vomiting, or prostration. Most cases are self-limiting with duration of illness varying from days to weeks. Illness can be more severe or life threatening in the young, old and immunocompromised (Blaser and Engberg, 2008). Of the 229,213 reported campylobacteriosis cases in the EU in 2015, 19,302 required hospitalization (31.2\%), and there were 59 deaths $(0.03 \%)$ (EFSA, 2016). In addition to the acute gastrointestinal disease, Campylobacter infection can be associated with a number of severe post-infectious complications. The most significant of these are believed to be GuillainBarré syndrome, reactive arthritis, irritable bowel syndrome, and inflammatory bowel disease (WHO, 2013). Many other sequelae are described including: pancreatitis, septic abortion, cholecystitis, nephritis, peritonitis, and myocarditis (Blaser and Engberg, 2008).

Broiler meat is considered to be the main source of human campylobacteriosis (Wilson et al., 2008; Sheppard et al., 2009), with undercooking or cross contamination likely to be the main routes of infection (EFSA, 2016). Consumption of undercooked or contaminated poultry meat is the largest single risk factor for sporadic infection, and other sources are described such as milk, water, other meats, and contact with pet or farm animals (Jacobs-Reitsma et al., 2008). Campylobacter colonization is widespread throughout poultry flocks. A recent European Food Safety Authority baseline survey found $75.3 \%$ of UK broiler flocks to be positive for Campylobacter (EFSA, 2010). In 2014, $76 \%$ of 497 chicken neck skin samples collected from UK slaughterhouses tested positive for Campylobacter (EFSA, 2015). Caecal or crop contents of broilers at slaughter can contain up to $10^{9}$ or $10^{5} \mathrm{CFU} / \mathrm{g}$, respectively, presenting a huge potential for the contamination of meat during evisceration (van Gerwe et al., 2010).

Resistance to oxidative stress is essential for C. jejuni colonization of the gastrointestinal tract (Hermans et al., 2011). Common sources of oxidative free radicals include the organisms' own respiratory metabolism, the ambient atmosphere in the extra-intestinal environment, and reactive oxygen species (ROS) generated by phagocytic or cytotoxic cells involved in the host immune response (Atack and Kelly, 2009). ROS damage DNA, proteins and lipids, limiting growth or killing the bacteria (Flint et al., 2016a). C. jejuni lacks several of the key oxidative stress response regulators identified in Escherichia coli, such as SoxRS, OxyR or Crp, and whereas E. coli possesses three separate superoxide dismutase and two catalase enzymes, C. jejuni encodes only one of each (Parkhill et al., 2000; Imlay, 2013; Flint et al., 2016b).

The three canonical enzymes involved in oxidative stress resistance in C. jejuni are superoxide dismutase, catalase, and alkyl hydroperoxide reductase. A superoxide dismutase $(\operatorname{sod} B)$ deficient mutant of $C$. coli had diminished environmental survival and decreased ability to colonize day old chicks (Purdy et al., 1999). A catalase (katA) deletion mutant C. jejuni demonstrated attenuated colonization fitness in a neonatal piglet model (Flint et al., 2012); however, a katA deficient C. coli had the same chick colonization characteristics as its parental strain (Purdy et al., 1999). Deletion of alkyl hydroperoxide reductase $(a h p C)$ in $C$. jejuni significantly reduced its ability to colonize day old chicks (Palyada et al., 2009). Other peroxiredoxins involved in resistance to oxidative stress are the thiol peroxidase Tpx and bacterioferritin comigratory protein BCP (Atack et al., 2008). Methionine sulfoxide reductase enzymes MsrA and MsrB protect C. jejuni from R- and S-methionine sulfoxides, which are produced from the oxidation of methionine. Deletion of $m s r A$ and $m s r B$ from $C$. jejuni resulted in increased sensitivity to ROS assays (Atack and Kelly, 2008). The DNA-binding protein from starved cells (Dps) has also been identified as a colonization factor for C. jejuni in a chick model and has been studied as a potential antigen candidate for a subunit anti-Campylobacter vaccine (Theoret et al., 2012). Other proteins involved in oxidative stress resistance of Campylobacter include cytochrome c peroxidases, quinone reductases, and DNA repair proteins (Atack and Kelly, 2009; Flint et al., 2016b). The response to oxidative stress is coordinated by a complex and overlapping network of regulators. These include the peroxide regulator (PerR) and ferric uptake regulator (Fur) (Palyada et al., 2009), the Campylobacter oxidative stress regulator (CosR) (Hwang et al., 2011), and the regulators of response to peroxide ( $\operatorname{RrpA}$ and $\operatorname{RrpB}$ ) (Gundogdu et al., 2015).

Campylobacter jejuni and C. coli are considered to be obligate microaerophilic bacteria, requiring oxygen concentrations between 2 and $10 \%$ for optimal growth (Kaakoush et al., 2007). The primary stress encountered in the extra-intestinal environment may be the ambient $21 \%$ oxygen (Atack and Kelly, 2009). Reduced sensitivity to ambient oxygen would confer superior environmental resistance, therefore increasing the likelihood of transmission between potential hosts. Improved survival of particular strains on chicken carcasses would be of obvious public health importance as the risks they pose would be higher than previously anticipated. In addition, if aerotolerance is related to oxidative stress resistance, this may render the organism less sensitive to free radicals produced by the host's immune response (Atack and Kelly, 2009), and therefore more pathogenic.

Campylobacter coli OR12 has been shown to be a highly successful colonizer of broiler chickens on organic and free-range farms (El-Shibiny et al., 2005). This strain is also capable of displacing other Campylobacter strains from pre-colonized chickens (El-Shibiny et al., 2007). An unusual characteristic of this strain is aerobic growth. Here we report adaptation of the strain to aerotolerance with the objective of determining whether the ability to withstand oxidative stress will modify the characteristics of C. coli OR 12 that make it a successful colonizer of broiler chickens. 


\section{MATERIALS AND METHODS}

\section{Bacterial Strains}

The Campylobacter strains used for this study were the chicken isolates C. coli OR12 (El-Shibiny et al., 2005), C. coli RM2228 (Fouts et al., 2005), C. jejuni HPC5 (Loc Carrillo et al., 2005), and the human clinical isolate C. jejuni NCTC 11168 (Parkhill et al., 2000).

\section{Aerobic Passage on Blood Agar (BA)}

Aerotolerant C. coli OR12 (Aer) was serially passaged by streaking on blood agar plates (BA; Oxoid) containing 5\% v/v defibrinated horse blood (TCS; Buckingham; UK) and incubating aerobically at $37^{\circ} \mathrm{C}$ for $2-7$ days. Intermediate stocks were archived at regular intervals by collecting confluent aerobic growth from a BA plate into freezer stock solution (15\% Glycerol, $85 \%$ Nutrient Broth No.2; Oxoid) and freezing at $-80^{\circ} \mathrm{C}$. Gram stains, using standard techniques, were performed at regular intervals to ensure contamination had not occurred. Four colonies were initially selected from the original growth and passaged independently. With the exception of pulsed-field gel electrophoresis (PFGE), all experiments were conducted using isolate $\mathrm{B}$, as it had qualitatively demonstrated the best growth in the early passages. The original wild type C. coli OR12 (WT) was maintained and stored microaerobically on BA.

\section{Quantitative Aerobic Growth or Survival on BA}

Blood agar 48 well plates were prepared by dispensing $1 \mathrm{ml}$ of BA to each well (Nunclon Delta; Nunc; Denmark). Inoculum suspensions of approximately $10^{7} \mathrm{CFU} / \mathrm{ml}$ of microaerobically grown C. coli RM2228, C. coli OR12 WT, C. coli OR12 Aer and aerobically grown $C$. coli OR12 Aer were prepared by suspending confluent growth from BA plates into PBS. Triplicate wells, representing biological replicates, were inoculated with $10 \mu \mathrm{l}$ of suspension (approximately $10^{5} \mathrm{CFU}$ ) per strain. Once dried, plates were incubated aerobically at $37^{\circ} \mathrm{C}$. After $0,6,24,30$, and $48 \mathrm{~h}$ of incubation, well contents were suspended by gentle repeated pipetting in maximum recovery diluent (MRD; Oxoid). Suspensions were enumerated on CCDA using a modification of the Miles Misra method (Miles et al., 1938). Briefly, decimal dilutions were performed in MRD, 5 replicate $10 \mu$ l droplets from up to six dilutions were plated on modified Campylobacter blood-free selective agar (CCDA; Oxoid) with 2\% agar. Once dry, plates were inverted, incubated microaerobically $\left(5 \% \mathrm{CO}_{2}, 5 \%\right.$ $\mathrm{O}_{2}, 2 \% \mathrm{H}_{2}, 88 \% \mathrm{~N}_{2}$ ) in a modular atmosphere controlled cabinet at $42^{\circ} \mathrm{C}$ (Don Whitley Scientific modified atmospheric cabinet, Shipley, UK) and examined after 24 and 48 h. Dilutions giving rise to between 3 and 30 colonies were selected, the sum of the 5 spots was multiplied by 20 , then multiplied by the inverse of the dilution factor to give the original CFU/ml.

\section{Qualitative Aerobic Growth on Solid Media (BA and CCDA)}

Suspensions of approximately $10^{8} \mathrm{CFU} / \mathrm{ml}$ of the following strains were prepared: microaerobically grown C. jejuni NCTC
11168; C. jejuni HPC5; C. coli RM2228; C. coli OR12 WT; C. coli OR12 Aer and aerobically grown C. coli OR12 Aer. Each inoculum was decimally diluted five times and one $10 \mu \mathrm{l}$ droplet of each dilution of each strain was dispensed onto two BA plates and two CCDA plates. Once dry, one of each was incubated microaerobically at $42^{\circ} \mathrm{C}$ and the other at $37^{\circ} \mathrm{C}$ aerobically. The microaerobic plates were examined after $48 \mathrm{~h}$ and the aerobic plates checked daily for 6 days.

\section{Survival in Aerobic Liquid Medium}

Sterile $250 \mathrm{ml}$ flasks containing $50 \mathrm{ml}$ Mueller-Hinton broth (MHB; Oxoid) were inoculated in triplicate to an approximate cell density of $10^{6} \mathrm{CFU} / \mathrm{ml}$ of microaerobically grown C. jejuni HPC5 and C. coli OR12 WT and aerobically grown C. coli OR12 Aer P25. These were incubated aerobically at $37^{\circ} \mathrm{C}$ with orbital shaking at $100 \mathrm{rpm}$. Samples were collected at 0, 2, 4, 6, 24, and $30 \mathrm{~h}$ and Campylobacter enumerated on CCDA as above.

\section{Growth Rate in Microaerobic Nutrient Broth No. 2}

Sterile $100 \mathrm{ml}$ flasks containing $30 \mathrm{ml}$ nutrient broth no.2 (NB2; Oxoid) were inoculated in triplicate with approximately $10^{4} \mathrm{CFU} / \mathrm{ml}$ of microaerobically grown C. coli RM2228 and C. coli OR12 WT and aerobically grown C. coli OR12 Aer P38. The flasks were incubated microaerobically in anaerobic gas jars using gas replacement $\left(7.3 \% \mathrm{CO}_{2}, 5.6 \% \mathrm{O}_{2}, 3.6 \% \mathrm{H}_{2}, 83 \% \mathrm{~N}_{2}\right)$ at $42^{\circ} \mathrm{C}$ with orbital shaking at $100 \mathrm{rpm}$ (John et al., 2011). Samples were collected and Campylobacter enumerated on CCDA at 0,2, 5 , and $8 \mathrm{~h}$.

\section{Motility Assays}

Motility was assessed using a motility agar assay and via membrane filtration. For the agar assay, inocula were prepared from freshly grown aerobic cultures of C. coli OR12 Aer or microaerobic cultures of C. coli OR12 WT. A pipette tip was used to collect a small plug of colony growth, which was then stabinoculated into the center of a motility agar (Mueller Hinton broth with $0.4 \% \mathrm{v} / \mathrm{v}$ agar) plate, without touching the bottom. Plates were incubated microaerobically at $42^{\circ} \mathrm{C}$ and examined after $48 \mathrm{~h}$.

For the membrane filtration assays, sterile nitrocellulose membrane filters with $0.45 \mu \mathrm{m}$ pore size and $47 \mathrm{~mm}$ diameter (Millipore) were aseptically placed in the center of BA plates. The centers of the filters were inoculated with $50 \mu \mathrm{l}$ of $C$. coli suspension taking care not to contaminate the surrounding agar. Once the liquid had passed through the filters, they were aseptically removed with sterile forceps. Plates were inoculated in duplicate, with one incubated microaerobically at $42^{\circ} \mathrm{C}$ and one aerobically at $37^{\circ} \mathrm{C}$.

\section{Peroxide Stress Assay}

Resistance to peroxide stress was performed using a method similar to that of Rodrigues et al. (2015). Glass universal containers, filled with $10 \mathrm{ml}$ of MRD containing $0,0.5$ or $1 \mathrm{mM} \mathrm{H}_{2} \mathrm{O}_{2}$ (Sigma; UK), were inoculated with identical optical densities of approximately $10^{8} \mathrm{CFU} / \mathrm{ml}$ microaerobically grown 
C. coli RM2228, C. coli OR12 WT, C. coli OR12 Aer P40 and aerobically grown $C$. coli OR12 Aer P40. Containers were incubated microaerobically at $42^{\circ} \mathrm{C}$ for $1 \mathrm{~h}$ with shaking at $100 \mathrm{rpm}$ followed by immediate enumeration of Campylobacter on CCDA as above. Three universals were inoculated per strain, representing independent biological replicates.

\section{Transmission Electron Microscopy}

Confluent BA cultures of microaerobically grown C. coli OR12 WT and aerobically grown C. coli OR12 Aer P37 were fixed in electron microscopy fixative (3\% glutaraldehyde in $0.1 \mathrm{M}$ cacodylate buffer) prior to centrifugation and resuspension in sterile water. Transmission electron microscopy (TEM) was performed at the Advanced Microscopy Unit, University of Nottingham. Samples were adsorbed onto copper Formvar/Carbon grids (AGS162-3; Agar Scientific) and negatively stained with $3 \%$ uranyl acetate. Grids were imaged using a Tecnai G12 biotwin TEM, run at $100 \mathrm{Kv}$, with a SIS megaview camera system and Gatan Microscopy Suite software (Gatan Inc.).

\section{Pulsed-Field Gel Electrophoresis}

Cell suspensions of four C. coli OR12 and aerotolerant derivatives were incorporated into agarose plugs, lysed and washed as described by Ribot et al. (2001). DNA plugs were cut into 2-mm slices and placed into $100 \mu \mathrm{l}$ of $1 \mathrm{x}$ SuRE/Cut Buffer A (Roche) and equilibrated at $25^{\circ} \mathrm{C}$ for $15 \mathrm{~min}$. This was replaced with $100 \mu \mathrm{l}$ of $0.2 \mathrm{U} / \mu \mathrm{l}$ SmaI (Roche) in the same buffer and incubated for $2 \mathrm{~h}$ at $25^{\circ} \mathrm{C}$. The plugs were incorporated in a gel consisting of $1 \%$ w/v Agarose (Bio-Rad) in TAE buffer. A 50-1000 kb DNA ladder (Lambda PFGE Ladder; New England BioLabs) was added as a marker. Electrophoresis was performed using a Bio-Rad CHEFDRII. The gel was stained in $50 \mu \mathrm{g} / \mathrm{ml}$ ethidium bromide in TAE buffer and visualized under UV light using the Gel Doc XR system with the Quantity One basic software, version 4.6.5 (Bio-Rad).

\section{Chicken Colonization Assay}

Ross 308 broiler chickens $(n=30)$ were obtained as day old chicks from a commercial hatchery (PD Hook; UK) and reared in biosecure conditions. Chicks were group reared in pens with a bedding of wood shavings and transferred to individual cages with an astroturf floor and environmental enrichment at 18 days of age. A 12-h light-dark cycle was followed for the duration of rearing. Temperatures were as outlined in the Code of Practice for the Housing and Care of Animals Bred, Supplied or Used for Scientific Purposes, with minor adjustments made as guided by bird thermoregulatory behavior. They received commercial broiler diets (starter, grower, and finisher) for the duration. Cloacal swabs were taken at day 18 and tested for Campylobacter by direct plating on CCDA and Salmonella by enrichment. At 21 days of age, 15 birds (Group 1) were gavaged with an estimated $10^{8} \mathrm{CFU}$ of C. coli OR12 WT and 15 birds (Group 2) with C. coli OR12 Aer P34. After 3 days, seven birds from each group were culled by parenteral barbiturate overdose, with the remaining eight birds culled at 7 days post challenge. Caecal contents were collected and enumerated for Campylobacter in triplicate as described above, with CCDA selective supplement (Oxoid) added to the medium. The membrane filtration assay was also performed on the $10^{-1}$ dilution of each caecal content sample, as described above. A secondary aerotolerance assay was performed on 24 or $48 \mathrm{~h}$ growth on the microaerobically incubated BA filtration plates. Growth was suspended in PBS and normalized to an $\mathrm{OD}_{600}$ of 0.05 , decimally diluted to $10^{-3}$ and a $10 \mu \mathrm{l}$ spot of each dilution inoculated to a BA plate which was incubated aerobically at $37^{\circ} \mathrm{C}$.

Power calculations were performed using the Spreadsheet for Sample Size Calculation (Brown, 2016). This indicated that seven birds were required per group to detect a $1 \log _{10} \mathrm{CFU} / g$ difference between groups with 95\% confidence and a standard deviation of $0.5 \log _{10} \mathrm{CFU} / \mathrm{g}$. One additional bird was added per group to allow for any unexpected mortality.

\section{Ethics Statement}

All experimental animal work was performed in accordance with UK and EU law. This study was approved by the Local Ethics Committee of the University of Nottingham and performed under Home Office license.

\section{Statistical Treatment of Data}

Viable counts were $\log _{10}$-transformed for analysis. Statistically significant differences $(p<0.05)$ were assessed using parametric (ANOVA) and non-parametric (Mann-Whitney $U$ ) tests through the statistical packages available within Minitab statistical software (Minitab Inc.).

\section{Whole Genome Sequencing and Analysis}

Genomic DNA was extracted from half of a confluent aerobically incubated BA plate of C. coli OR12 Aer P43. DNA was isolated using the GenElute Bacterial Genomic DNA Kit (Sigma-Aldrich) according to manufacturer's instructions. Sequencing was performed using the MiSeq platform at Northumbria University. The sequence reads were aligned to the assembled sequence of C. coli OR12 (NCBI RefSeq NZ_CP013733.1) and appear in the database as CcOR12aero (NCBI RefSeq NZ_CP019977). DNA sequence variants were detected using the variant detector within CLC Genomics workbench 9.01 (Qiagen Bioinformatics). Candidate high probability nucleotide sequence variants detected were confirmed by careful inspection of the sequence reads, and any predicted changes to the protein encoding sequences confirmed using the Artemis genome browser (Rutherford et al., 2000). The predicted protein sequences were further compared with wild-type $C$. coli OR12 sequences, and with other similar protein sequences using the protein basic local alignment tool, BLASTP mounted on the NCBI website ${ }^{1}$. Nucleotide BLAST ${ }^{1}$ was used to identify orthologs of genes with known roles in Campylobacter oxidative stress resistance. Protein sequences of gene products in C. jejuni NCTC11168 and $C$. coli OR12 were compared using the protein BLAST function.

\footnotetext{
${ }^{1}$ https://blast.ncbi.nlm.nih.gov/Blast.cgi
} 


\section{RESULTS}

\section{Growth and Survival Characteristics of Aerotolerant C. coli OR12}

For the initial early passages, growth of aerotolerant C. coli OR12 was limited to the heavily inoculated region of the BA plate. After approximately 15 aerobic passages, growth of single colonies became visible within 2-5 days of aerobic incubation at $37^{\circ} \mathrm{C}$. Gram staining confirmed typical Campylobacter morphologies with variable populations of Gram-negative spirals, filamentous, and coccoid forms. Approximately $10^{5} \mathrm{CFU}$ of C. coli RM2228, wild type C. coli O12 (WT), aerotolerant C. coli OR12 (Aer) passage (P) 32, and a microaerobic preparation of $C$. coli OR12 Aer P32 were inoculated onto BA microplates and aerobically incubated at $37^{\circ} \mathrm{C}$. No changes in viable count were apparent after 6 h; however, by 24 h the C. coli RM2228 and C. coli OR12 had declined to below the limit of detection ( $\left.1.3 \log _{10} \mathrm{CFU}\right)$. In contrast, both the C. coli OR12 Aer P32 and the microaerobically prepared C. coli OR12 Aer P32 had grown by more than $1 \log _{10}$ after $24 \mathrm{~h}$ and continued to grow, with viable counts of 7.4 and $8.3 \log _{10}$ CFU, respectively, by $48 \mathrm{~h}$ (Figure 1).

Differences between aerobic and microaerobic growth were examined on BA and CCDA plates that had been inoculated with six decimal dilutions of a panel of six Campylobacter strains. These included C. coli OR12 WT along with aerobically and microaerobically prepared $C$. coli OR12 Aer P38. After $48 \mathrm{~h}$ of microaerobic incubation, single colonies were present at the highest dilution $\left(10^{-5}\right)$ of all strains on BA and CCDA, correlating with viable count ranges of between $5.7 \times 10^{7}$ and $2.5 \times 10^{8} \mathrm{CFU} / \mathrm{ml}$ for the initial inocula. Viable counts of all strains were within $1 \log _{10}$ of each other (Figure 2). After 6 days of aerobic incubation, single colonies were present down to the
$10^{-4}$ dilution of both aerobically and microaerobically prepared C. coli Aer P38 on BA and CCDA. No aerobic growth was present from any of the other strains examined (Figure 2). The growth rates of C. coli RM2228, C. coli OR12 WT and C. coli O12 Aer $\mathrm{P} 38$ were assessed by microaerobic incubation at $42^{\circ} \mathrm{C}$ in nutrient broth no. 2. Viable counts are shown in Supplementary Figure 1. Exponential growth rates for each strain were calculated in the logarithmic growth phase; rates were not significantly different between strains.

Aerobic survival in liquid medium was assessed by inoculating Mueller Hinton broth with an estimated $6 \log _{10} \mathrm{CFU} / \mathrm{ml}$ and incubating at $37^{\circ} \mathrm{C}$. Viable counts are presented in Supplementary Figure 2. Following $6 \mathrm{~h}$ of aerobic incubation, the C. jejuni HPC5, C. coli OR12 WT and C. coli OR12 Aer had declined by $0.17,0.23$, and $0.42 \log _{10} \mathrm{CFU} / \mathrm{ml}$, respectively. By 24 h, both C. coli OR12 strains and two of the C. jejuni HPC5 flasks had declined to below the limit of detection $\left(1.3 \log _{10}\right.$ $\mathrm{CFU} / \mathrm{ml}$ ) and one C. jejuni HPC5 flask had a viable count of $2.4 \log _{10} \mathrm{CFU} / \mathrm{ml}$. Despite differences in the starting inocula, aerotolerant $C$. coli OR12 did not demonstrate superior survival compared to the wild type and C. jejuni HPC5.

\section{Aerotolerant C. coli OR12 Remain Motile in Atmospheric Oxygen}

Motility assays were performed to determine whether serial aerobic passage resulted in reduced motility. The C. coli OR12 WT strain was tested together with C. coli OR12 Aer P22 and P50. All isolates demonstrated motility, with diameters of growth of 35,34 and $33 \mathrm{~mm}$, respectively, therefore aerotolerance was not associated with any loss of motility.

Owing to their highly energetic corkscrew-like motility campylobacters can traverse membrane filters that would exclude

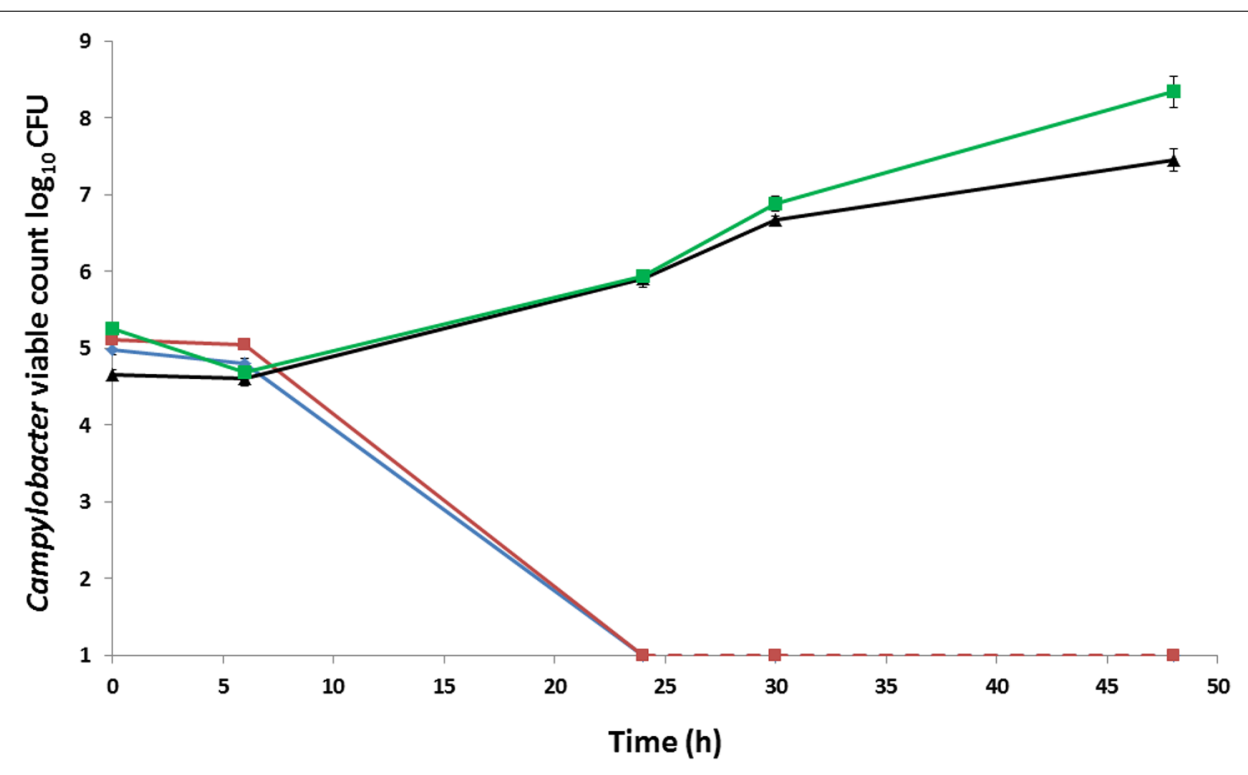

FIGURE 1 | Growth and survival of Campylobacter coli strains incubated aerobically on BA at $37^{\circ} \mathbf{C}$. C. coli RM2228 (blue), C. coli OR12 WT (red), aerobically prepared C. coli OR12 Aer P32 (black), microaerobically prepared C. coli OR12 Aer P32 (green). Error bars represent standard deviations from three biological replicates. Results are representative of three separate experiments. The limit of detection was $1.3 \log _{10} \mathrm{CFU}$. 

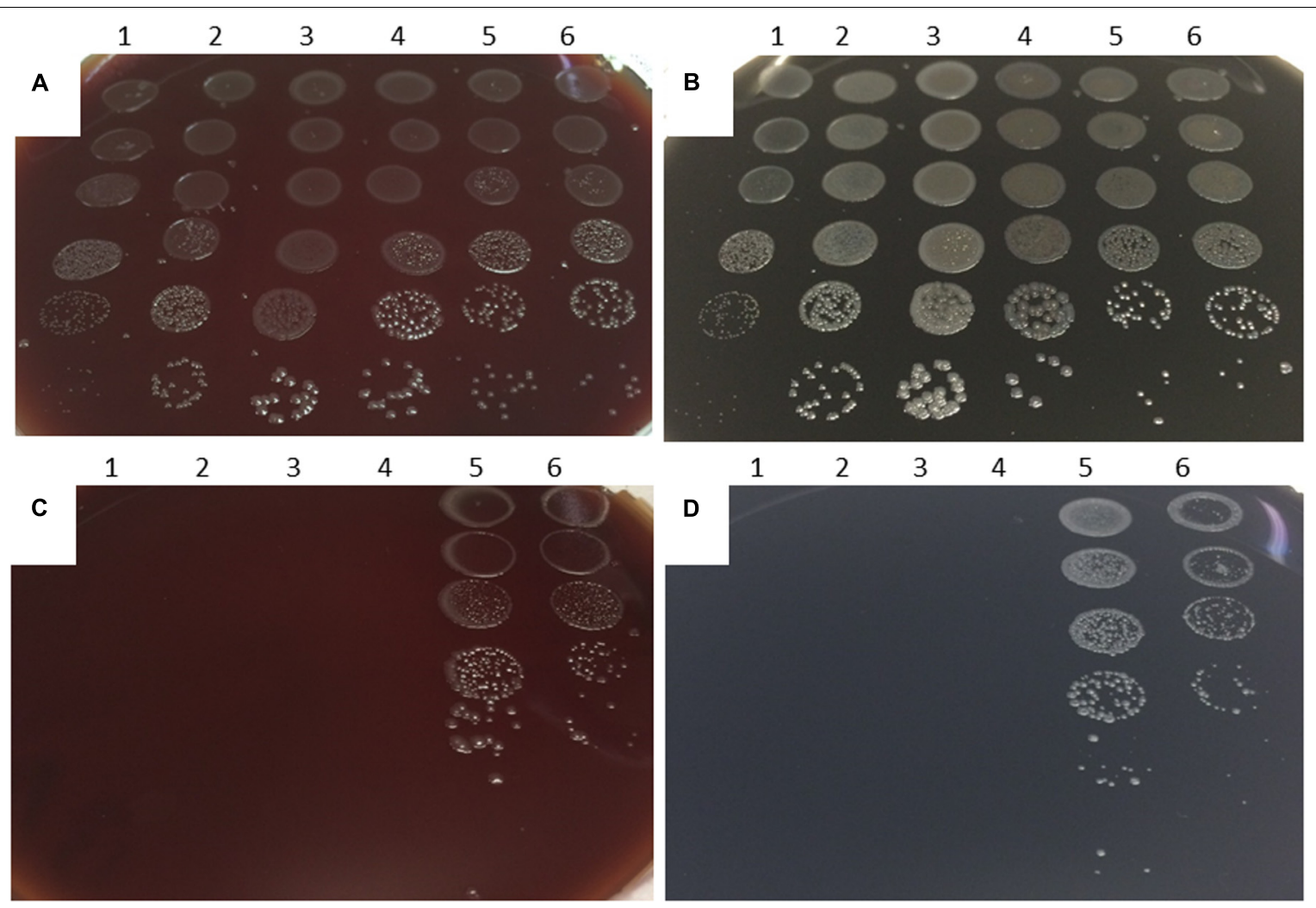

FIGURE 2 | Microaerobic and aerobic growth of Campylobacter on BA and CCDA. Decimal dilutions of suspensions of: (1) C. jejuni NCTC 11168; (2) C. jejuni HPC5; (3) C. coli RM2228; (4) C. coli OR12 WT; (5) C. coli OR12 Aer P38 (aerobic prep); (6) C. coli OR12 Aer P38 (microaerobic prep) inoculated onto:

Microaerobically incubated BA (A) and CCDA (B) after $48 \mathrm{~h}$. Aerobically incubated BA (C) and CCDA (D) after 6 days.

other bacteria, a property that has been used as a basis for their isolation from several animal sources (Moreno et al., 1993). Filtration was therefore used to examine the motility of aerotolerant C. coli OR12. Duplicate BA plates with centered nitrocellulose membrane filters were inoculated with $50 \mu \mathrm{l}$ of suspensions containing $7.6 \log _{10} \mathrm{CFU} / \mathrm{ml}$ C. coli OR12 WT or $7.6 \log _{10} \mathrm{CFU} / \mathrm{ml}$ C. coli OR12 Aer P38. One of each was incubated microaerobically at $42^{\circ} \mathrm{C}$ and one of each aerobically at $37^{\circ} \mathrm{C}$. After $24 \mathrm{~h}$, heavy growth was present in the center of the filtered regions of both plates incubated microaerobically. After 4 days, growth was evident on the aerotolerant $C$. coli OR12 incubated aerobically, with confluent colonies by 5 days. No growth was noted on the wild type C. coli OR12 incubated aerobically. Under aerobic conditions aerotolerant $C$. coli OR12 remained motile and metabolically active to be able to translocate the membrane filter.

\section{Aerotolerant C. coli OR12 Demonstrates Increased Peroxide Stress Resistance}

To measure oxidative stress resistance, suspensions containing between $7.3 \log _{10}$ and $7.8 \log _{10} \mathrm{CFU} / \mathrm{ml}$ C. coli RM2228, C. coli OR12 WT, aerobically prepared C. coli OR12 Aer P40 and microaerobically prepared C. coli OR12 Aer P40 were exposed to different concentrations of $\mathrm{H}_{2} \mathrm{O}_{2}$. Marked differences in survival were apparent between aerobically prepared aerotolerant C. coli OR12 and the other microaerobically prepared strains (Figure 3).

\section{Transmission Electron Microscopy}

Transmission electron micrograph (TEM) images are shown in Figure 4. Both wild type and aerotolerant $C$. coli OR12 contained populations of typical spiral cells as well as filamentous and coccoid forms.

\section{Colonization of Chickens}

Colonization gavage doses were titrated at $1.38 \times 10^{8} \mathrm{CFU}$ for group 1 (C. coli OR12 WT) and $3.4 \times 10^{7} \mathrm{CFU}$ for group 2 (C. coli OR12 Aer P34). Caecal Campylobacter counts at 3 and 7 days post gavage are shown in Figure 5. All the birds that received C. coli OR12 WT became colonized, with mean caecal Campylobacter counts of $8.71( \pm 0.2)$ and $8.57( \pm 0.3) \log _{10} \mathrm{CFU} / \mathrm{g}$ at 3 and 7 days post gavage, respectively. Of the birds challenged with C. coli OR 12 Aer, only $2 / 7$ had detectably colonized by 3 days and $6 / 8$ by 8 days. Birds with no Campylobacter growth were considered $\leq 2.3 \log _{10} \mathrm{CFU} / \mathrm{g}$, which was the limit of detection. The difference in caecal Campylobacter counts between the wild type and aerotolerant strain was significant at 3 days post challenge $(p=0.006)$, but not significant by day $7(p=0.0659)$ as determined by the two-tailed Mann-Whitney $U$ test. Standard 


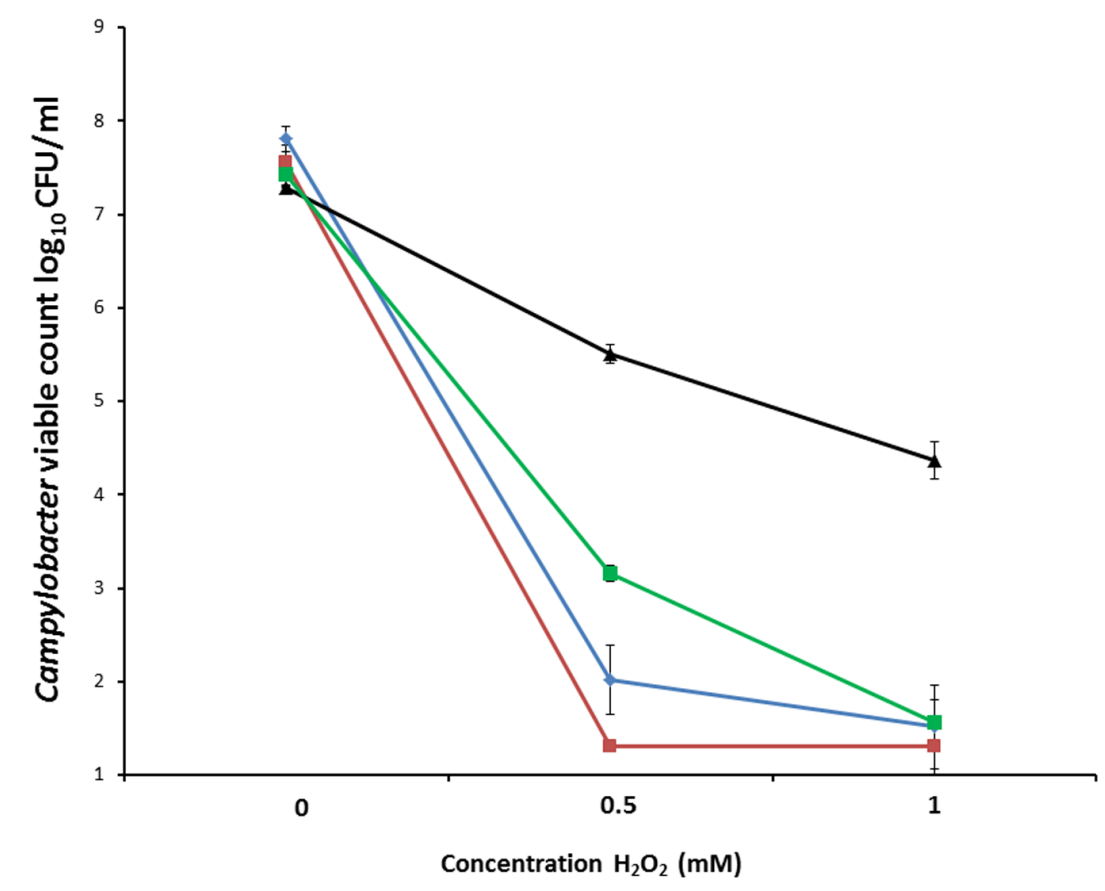

FIGURE 3 | Survival of C. coli in differing concentrations of hydrogen peroxide. C. coli RM2228 (blue), C. coli OR12 WT (red), aerobically prepared C. coli OR12 Aer P40 (black), microaerobically prepared C. coli OR12 Aer (green). Cultures were incubated for $1 \mathrm{~h}$ at $42^{\circ} \mathrm{C}$ under microaerobic conditions. Error bars represent standard deviations from three biological replicates.

deviations for individual bird Campylobacter counts generated by triplicate technical replicates were all below $0.19 \log _{10}$ $\mathrm{CFU} / \mathrm{g}$.

All birds from which Campylobacter was detected on CCDA also yielded positive growth on microaerobically incubated membrane filtered BA plates. Colony morphology was consistent with Campylobacter (Supplementary Figure 3) and Gram stains provided confirmation. Of the aerobically incubated membrane filtration BA plates, growth was detected from one of the C. coli OR12 Aer challenged birds at 3 days post challenge and from 3 birds at 7 days post challenge. No growth was detected on any of the aerobically incubated membrane filtered BA plates from wild type challenged birds.

Secondary aerotolerance assays were performed on the campylobacters isolated by microaerobic membrane filtration. All C. coli isolated from birds challenged with C. coli OR12 Aer P34 retained their aerotolerance. Supplementary Figure 3A shows the serially diluted and aerobically incubated C. coli isolated from the two birds 3 days post challenge with C. coli OR12 Aer P34. No such growth was noted from isolates recovered from the seven birds challenged with the wild-type strain.

\section{Whole Genome Sequencing and Analysis}

Macrorestriction patterns of wild type C. coli OR12 and 4 aerobically passaged isolates determined by PFGE were identical to each other (Supplementary Figure 4) and to the original isolate described by El-Shibiny (2006). The development of aerotolerance is not associated with any significant DNA insertion, deletion or genome rearrangement. Therefore whole genome sequencing was performed to examine the occurrence of any point mutations.

A total of 23 mutations were noted between the wild type and aerotolerant $C$. coli OR12 sequences. There were 11 single nucleotide substitutions, 2 nucleotide insertions and 10 insertions or deletions within homopolymeric GC tracts. The DNA changes within homopolymeric GC tracts are summarized in Table $\mathbf{1}$ and all others in Table 2. Three of the mutations were silent and one was within a non-coding region. All the predicted protein sequences were analyzed with BLASTP to confirm the protein identity and to determine if the changes induced were present in other similar sequences.

\section{Phosphoglycerate Kinase}

Gene pgk, which encodes the 400 amino acid protein phosphyglycerate kinase is located between nucleotides 380840382042. BLASTP analysis of the amino acid sequence identified many C. coli and C. jejuni sequences with 99-100\% coverage and identity. Aerotolerant $C$. coli OR12 has an A insertion at nucleotide position 380,879 , which results in a frame shift at residue 19 and early protein termination at 21 amino acids. The entire amino acid sequence of the mutant protein is: MSDIISIKDIDLSKKKSFYKM. BLASTP analysis of this sequence did not find any sequences of similar length.

\section{Biotin Attachment Protein/Pyruvate Carboxylase B}

Gene cfiA encodes a 597 amino acid product, which was initially annotated as 2-oxoglutarate carboxylase. Subsequent BLASTP 


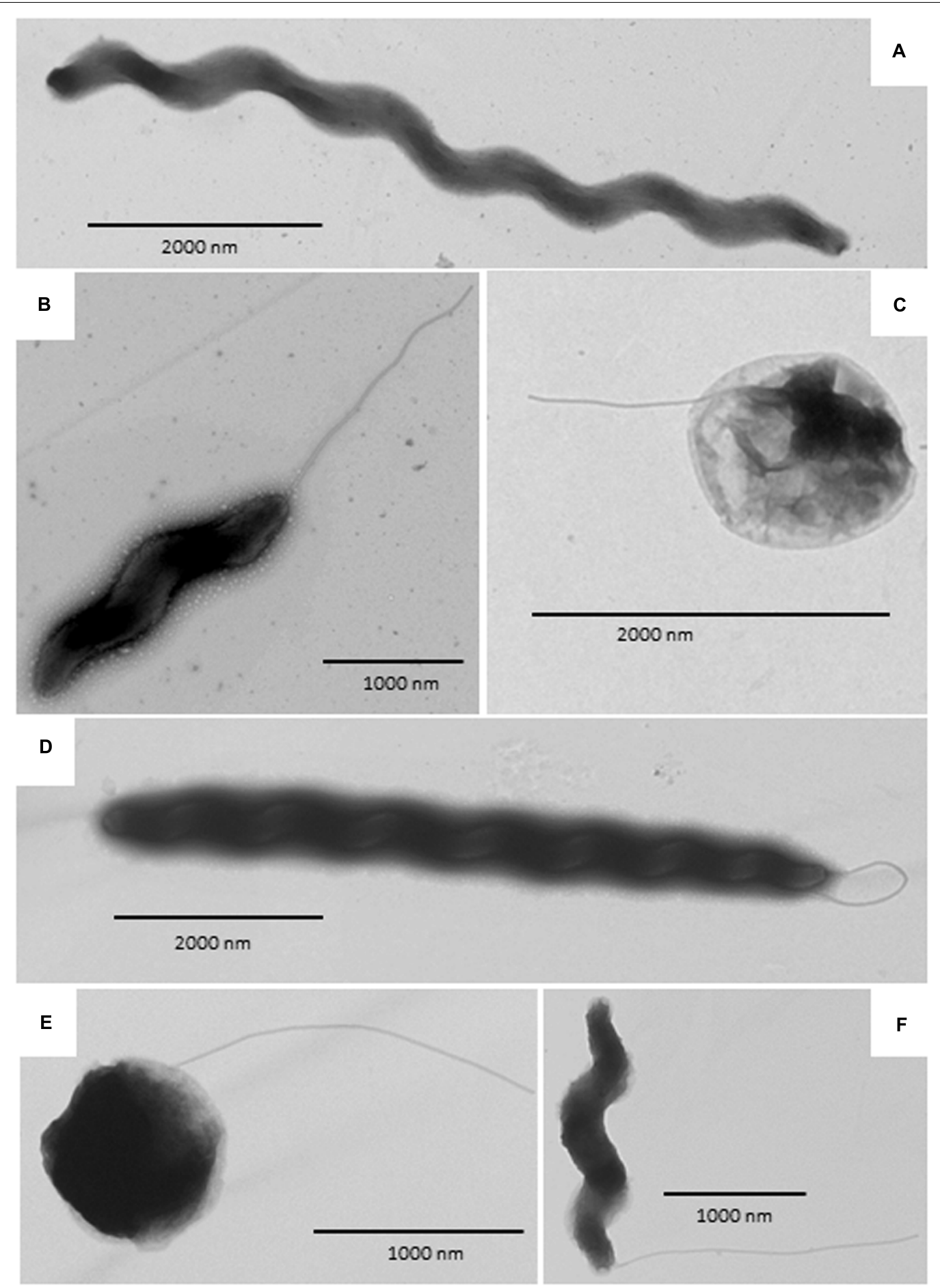

FIGURE 4 | Transmission electron microscopy images of C. coli OR12. (A-C)Rrepresent wild type and (D-F) aerotolerant C. coli OR12. (A) Filamentous form; (B) Spiral form with flagellum at the poles; (C) Coccoid form with flagellum; (D)Filamentous form with flagella; (E) Coccoid form with flagellum; (F) Spiral form with single polar flagellum.

searches identified $99 \%$ amino acid sequence identity with many C. coli biotin attachment proteins. The sequence also shares identity with several conserved domains, including phosphate binding superfamily, carboxylase, carboxylase interaction sites and a biotinylation site. The sequence of aerotolerant $C$. coli OR12 has a $\mathrm{T}$ insertion at position 837,990 . This results in a frame shift and premature termination at residue 419. BLASTP analysis of the mutant sequence identified no similar sequences of identical 


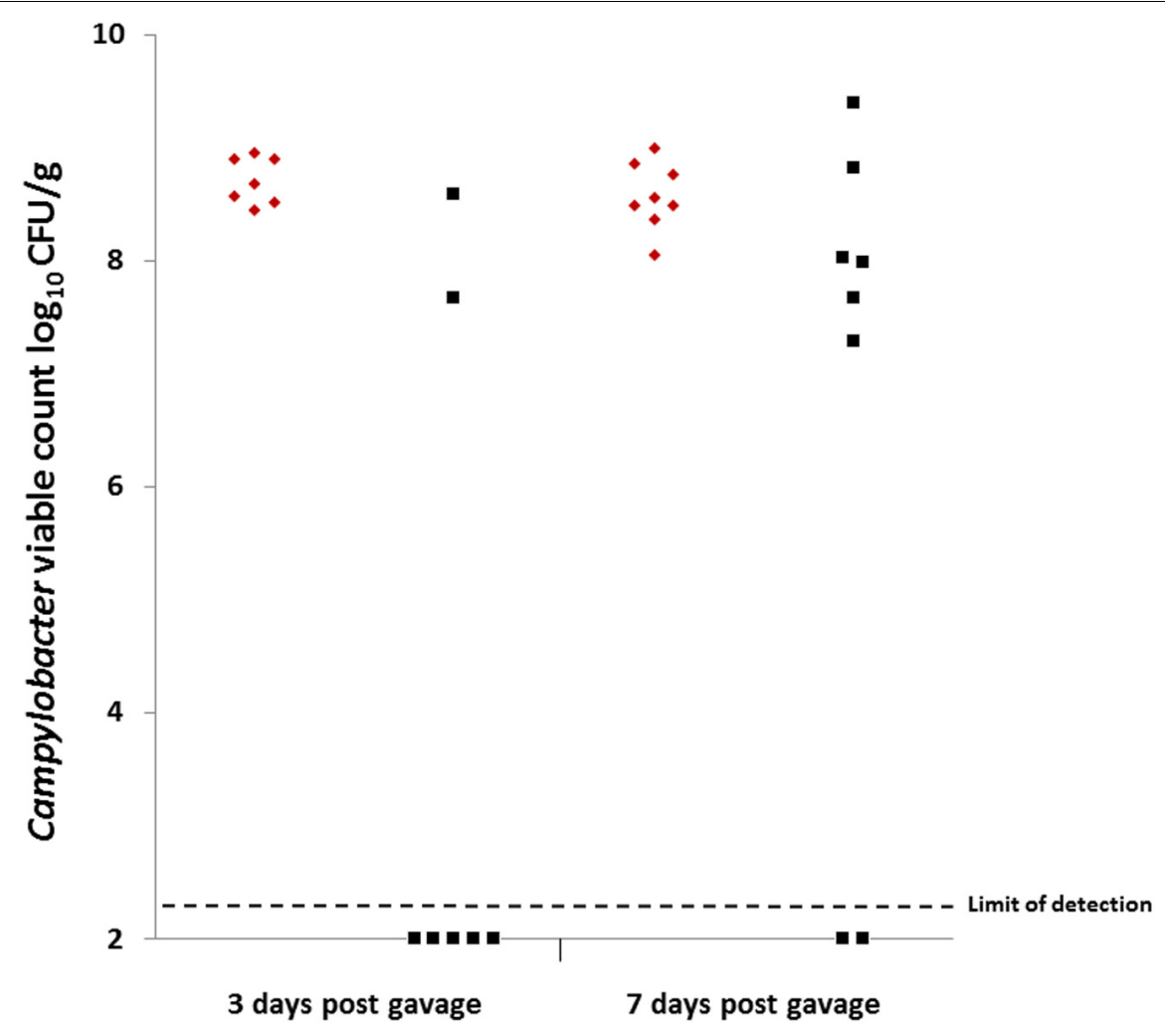

FIGURE 5 | Caecal Campylobacter counts from chickens challenged with, C. coli OR12 WT and Aer P34 at 3 and 7 days post challenge. C. coli WT (red diamonds), C. coli OR12 Aer (black squares). Counts between wild type and aerotolerant strains were statistically significant 3 days post challenge ( $p=0.006)$ but not at 7 days $(p=0.0659)$ using Mann-Whitney $U$ tests.

length, and lacked part of the carboxylase domain as well as the entirety of the biotinylation and carboxylase interaction sites.

\section{PseD/Motility Accessory Factor}

Nucleotide position 455,044 lies within a poly-C tract. In wild type C. coli OR 12 this tract is composed of $10 \mathrm{Cs}$ in $83 \%$ of the sequence reads, and 9 Cs in $7 \%$ of the sequences. The aerotolerant C. coli OR12 sequence has become fixed with $100 \% 9$ Cs at this location. This position is within genes ATE51_00982 and ATE51_00984. In the $10 \mathrm{C}$ configuration, the reading frame ATE51_00982 encodes a 593 amino acid protein that shares high sequence identity with PseD proteins (97 to 100\% identity) from

TABLE 1 | Mutations in aerotolerant Campylobacter coli OR12 present within homopolymeric tracts.

\begin{tabular}{|c|c|c|c|c|c|}
\hline Position & $\begin{array}{l}\text { Consensus } \\
\text { nucleotide change }\end{array}$ & Gene/CDS & Product & Change & AA change \\
\hline 455044 & $C(-)$ & ATE51_00892 & PseD protein/Motility accessory factor & Premature termination & Y5 FS \\
\hline 455044 & $C(-)$ & ATE51_00894 & Hypothetical protein/Motility accessory factor & Fusion to ATE51_00892 & V60 FS \\
\hline 565851 & $\mathrm{G}(-)$ & $h \times u A$ & $\begin{array}{l}\text { Heme/hemopexin-binding protein precursor/filamentous } \\
\text { hemagglutinin }\end{array}$ & Premature termination & M19 FS \\
\hline 828036 & $\mathrm{CC}(+)$ & pepD & Aminoacyl-histidine dipeptidase & Premature termination & I186 FS \\
\hline 483767 & $\mathrm{G}(-)$ & ATE51_00954 & Hypothetical protein/carbonic anhydrase (incomplete) & C-terminal change & G196 FS \\
\hline 484990 & $\mathrm{G}(-)$ & ATE51_00958 & Hypothetical protein/carbonic anhydrase (incomplete) & Premature termination & S197 FS \\
\hline 1158035 & $\mathrm{CC}(-)$ & ATE51_02354 & Hypothetical protein/carbonic anhydrase & C-terminal change & G189 FS \\
\hline 64160 & $\mathrm{CC}(-)$ & ATE51_00096 & Iron-binding protein & C-terminal DR deletion & D240 FS \\
\hline 465041 & $\mathrm{CC}(-)$ & ATE51_00914 & Methyltransferase & C-terminal VL $\rightarrow$ VKL & V88 FS \\
\hline 366720 & $\mathrm{GG}(-)$ & $y j j G$ & Pyrimidine 5'nucleotidase/uridine kinase (incomplete) & C-terminal $V \rightarrow I G$ & V194 FS \\
\hline 422439 & $\mathrm{GG}(-)$ & Non-coding & N/A & $\mathrm{N} / \mathrm{A}$ & N/A \\
\hline
\end{tabular}

Consensus nucleotide changes represent differences in the aerotolerant C. coli genome sequence by addition (+) or deletion (-) compared to the wild type sequence. FS indicates the change results in a frame shift. 
TABLE 2 | Mutations in aerotolerant C. coli OR12 not within homopolymeric tracts.

\begin{tabular}{|c|c|c|c|c|c|}
\hline Position & $\begin{array}{l}\text { Nucleotide } \\
\text { change }\end{array}$ & Gene/CDS & Product & Change & AA change \\
\hline 380879 & $A+$ & pgk & Phosphoglycerate kinase & Frame shift and premature termination & V19 frameshift \\
\hline 837990 & $\mathrm{~T}+$ & $c f i A$ & $\begin{array}{l}\text { Biotin attachment protein/Pyruvate } \\
\text { carboxylase B subunit }\end{array}$ & Frame shift and premature termination & D419 frameshift \\
\hline 116044 & $\mathrm{C} \rightarrow \mathrm{T}$ & nhaA1 & $\mathrm{Na} / \mathrm{H}$ antiporter & Single aa substitution & $A 166 \rightarrow V$ \\
\hline 358671 & $\mathrm{C} \rightarrow \mathrm{T}$ & epsE & Glycosyl transferase family A & Single aa substitution & $\mathrm{T} 367 \rightarrow 1$ \\
\hline 615756 & $A \rightarrow G$ & $m n m G$ & $\begin{array}{l}\text { tRNA uridine 5-carboxymethylaminomethyl } \\
\text { modification enzyme GidA }\end{array}$ & Single aa substitution & $\mathrm{T} 368 \rightarrow \mathrm{A}$ \\
\hline 56663 & $\mathrm{G} \rightarrow \mathrm{A}$ & TyрA/BipA & GTP- binding protein & Single aa substitution & $\mathrm{T} 210 \rightarrow 1$ \\
\hline 168195 & $\mathrm{C} \rightarrow \mathrm{T}$ & sat & Sulfate adenylyltransferase & Single aa substitution & $A 290 \rightarrow V$ \\
\hline 1346838 & $A \rightarrow G$ & ATE51_02794 & $\begin{array}{l}\text { Hypothetical protein/VgrG type VI secretion } \\
\text { protein (incomplete) }\end{array}$ & Single aa substitution & $\mathrm{D} 19 \rightarrow \mathrm{G}$ \\
\hline 1722359 & $\mathrm{G} \rightarrow \mathrm{A}$ & panc & Pantoate beta alanine ligase & Single aa substitution & $\mathrm{E} 216 \rightarrow \mathrm{K}$ \\
\hline 1948793 & $\mathrm{C} \rightarrow \mathrm{T}$ & $\operatorname{infB}$ & Translation initiation factor IF-2 & Single aa substitution & $\mathrm{R} 720 \rightarrow \mathrm{H}$ \\
\hline 615323 & $\mathrm{C} \rightarrow \mathrm{T}$ & $m n m G$ & $\begin{array}{l}\text { 5-carboxymethylaminomethyl modification } \\
\text { protein }\end{array}$ & Silent & None \\
\hline 1205648 & $\mathrm{~T} \rightarrow \mathrm{C}$ & $x e r D$ & Recombinase & Silent & None \\
\hline 1205660 & $A \rightarrow G$ & xerD & Recombinase & Silent & None \\
\hline
\end{tabular}

many other C. coli and C. jejuni data base entries. The sequence also has $90 \%$ coverage and $71 \%$ identity with the sequence of PseD from C. jejuni NCTC 11168, which is involved in flagellar modification (McNally et al., 2006). The 9 C configuration, present in the minority of the wild type and all of the aerotolerant C. coli OR12 sequences, results in a frame shift at the fifth amino acid residue and protein termination at 21 amino acids. The 10 C configuration of gene ATE51_00984 encodes a 60 amino acid protein. However, the $9 \mathrm{C}$ configuration results in a frame shift after 60 amino acid residues and fusion with ATE51_00982 resulting in a 648 amino acid protein, which has 99\% coverage and $72 \%$ identity with the PseD from C. jejuni NCTC 11168.

\section{Filamentous Haemagglutinin}

Gene $h x u A$ encodes a 1063 amino acid protein that was annotated as a heme/hemopexin binding precursor. However, BLASTP analysis of the protein sequence identified many C. coli filamentous haemagglutinin proteins with over $90 \%$ coverage and identity. The amino acid sequence also contains a conserved $N$-terminal haemagglutinin domain, conserved in many proteins with haemagglutination or haemolysin activity. Nucleotide 565,851 lies within a poly-G tract. Of the wildtype C. coli OR12 sequences, $96 \%$ of contained $10 \mathrm{Gs}$ and $4 \%$ contained 9 Gs. Aerotolerant C. coli OR12 sequences were composed of $75 \%$ containing $9 \mathrm{Gs}$ and $25 \%$ containing $10 \mathrm{Gs}$. The $10 \mathrm{G}$ configuration results in encoding of the full 1063 amino acid protein; however, the $9 \mathrm{G}$ configuration results in a frame shift at residue 19 and immediate termination.

\section{PepD}

Gene $p e p D$ is a disrupted pseudogene in the majority of C. coli OR12 sequences. Nucleotide 828,036 lies within a polyC tract. The wild-type $C$. coli OR12 sequences are composed of: $91 \%$ containing 8 Cs and $9 \%$ containing 9 Gs. Aerotolerant
C. coli OR12 sequences are composed of: $77 \%$ containing 10 Gs and $23 \%$ containing 9 Gs. The 8 G configuration, which represents the majority of wild-type sequences, encodes a 221 amino acid protein that shares high sequence identity $(83 \%$ coverage and $100 \%$ identity) with many C. coli aminoacylhistidine dipeptidase proteins that are significantly longer. The $9 \mathrm{C}$ configuration, present in the minority of wild-type and aerotolerant sequences, encodes a 423 amino acid protein, which has high sequence identity (99\%) with other C. coli aminoacylhistidine dipeptidase sequences of the same length. The sequence also shares similarity with a conserved zinc peptidase like superfamily domain. The $10 \mathrm{C}$ configuration, present in the minority of aerotolerant sequences, encodes a 194 amino acid protein, which is identical to the $8 \mathrm{G}$ product apart from the final 9 amino acid residues. Of the protein sequences that show high similarity with the $8 \mathrm{G}$ and $10 \mathrm{G}$ products, none ended in the same location or with similar C-terminal amino acid residues.

\section{Carbonic Anhydrases}

The first 192 amino acids of this predicted 205 amino acid protein product of gene ATE51_00954 share 67\% identity with the first portion of the adjacent ATE51_00952 and nearby ATE51_00958, both of which are annotated as putative carbonic anhydrases. BLASTP analysis indicates $100 \%$ identity with several 196 amino acid proteins in C. coli and C. jejuni also similarly annotated as carbonic anhydrases. Aerotolerant C. coli OR12 has a single $G$ homopolymer deletion at position 483,767 which causes a frame shift at the G196 residue, replacing residues GGHMEDTYLYS with GVIWRIPIFTHSKDSSLVCGWRD at the carboxyl terminus. BLASTP analysis of the 217 aa mutant configuration identified $88-90 \%$ identity with one C. jejuni and one $C$. coli hypothetical protein of 218 aa length, however, the majority of entries demonstrating sequence similarity were for 403-404 aa carbonic anhydrases. 
Gene ATE51_00958 encodes a 405 aa protein, annotated as carbonic anhydrase with $97-100 \%$ identity with C. jejuni and $C$. coli carbonic anhydrase proteins of identical length. Aerotolerant $C$. coli OR12 has a G deletion at nucleotide 484,990 which induces a frame shift at residue 197, altering the aa sequence with a stop at 201 aa. BLASTP analysis of the mutant sequence revealed $97 \%$ identity with two C. coli hypothetical proteins of identical length, one of which was in C. coli RM2228.

Nucleotide position 1,158,035 lies within a homopolymeric tract, with wild-type C. coli OR12 sequences consisting of: $90 \%$ with $10 \mathrm{Cs} ; 7 \%$ with $9 \mathrm{Cs}$ and $3 \%$ with $11 \mathrm{Cs}$. Aerotolerant C. coli OR12 sequences consist of: $85 \%$ with 8 Cs and $15 \%$ with 9 Cs. The original annotation of ATE51_02354 indicated a hypothetical protein; however, a more recent annotation of the $C$. coli OR12 genome within the Genbank database classified the region as a disrupted carbonic anhydrase pseudogene. The $10 \mathrm{C}$ configuration, representing $90 \%$ of wild-type C. coli OR12 sequences, results in a predicted 190 aa hypothetical protein, with many highly similar hypothetical proteins in the database. One entry suggests high sequence identity with a partial carbonic anhydrase in C. coli (entry KQH53282.1). The $8 \mathrm{C}$ configuration, representing $85 \%$ of aerotolerant $C$. coli OR12 sequence reads, results in a frame shift and exchange of the terminal GY residue for VLERFCLEIHMADI. This arrangement is also present in several similar sequences in the database, again mostly annotated as hypothetical proteins. The $9 \mathrm{C}$ configuration, present in $7 \%$ of wild type and $15 \%$ of aerotolerant C. coli OR12 sequence reads results in a frame shift and fusion with the next open reading frame, ATE51_02352, resulting in a 408 amino acid protein. This sequence is also present in the database, with all similar entries annotated as hypothetical proteins. Residues 194-408 have a 99\% similarity to a putative carbonic anhydrase domain in C. jejuni DFVF1099 (Takamiya et al., 2011). The 11 C configuration (3\% of wild-type $C$. coli OR12 sequence reads) results in a frame shift similar to the $8 \mathrm{C}$ configuration, but an additional $G$ residue is retained; only the terminal $Y$ amino acid residue is exchanged for the residues VLERFCLEIHMADI. This configuration is less frequently reported in the database than that of the $8 \mathrm{C}$ variant. All variants result in high similarity with the conserved domain DUF2920, a bacterial domain of unknown function. The three putative carbonic anhydrase protein sequences above share significant identity at their $\mathrm{N}$-termini, as demonstrated by the alignment in Supplementary Figure 5.

\section{Oxidative Stress Resistance Genes}

Genes involved in Campylobacter oxidative stress resistance, as cataloged in a recent review by Flint et al. (2016b), were identified in C. coli OR12 and the corresponding protein sequences compared with orthologs from C. jejuni NCTC11168. These data are presented in Supplementary Table 1. All the functional genes cataloged by Flint et al. (2016b) could be identified with the exception of those encoding the regulators of response to peroxide $\mathrm{A}$ and $\mathrm{B}(\operatorname{rrp} A$ and $\operatorname{rrp} B)$. No significant sequence similarity to the latter two genes could be identified in the sequences of C. coli OR12 or C. coli RM1875 or C. coli 15-537360.

\section{DISCUSSION}

Aerotolerance of Campylobacter spp. may represent a survival adaptation, potentially allowing superior environmental persistence and therefore improved opportunities for colonization of new hosts. The ability to withstand atmospheric oxygen is also relevant to survival on chicken meat and is therefore of public health significance. Aerotolerance is related, at least in part, to oxidative stress resistance which has been shown to be a colonization factor for chickens and a virulence factor for human infection (Hermans et al., 2011; Bolton, 2015; Flint et al., 2016a).

Aerotolerance following aerobic passage of C. coli OR12 was demonstrated by aerobic growth on both BA and CCDA. Similar viable counts of wild type C. coli OR12 and C. coli RM2228 were inoculated to BA, but they failed to grow and had declined below limit of detection by $24 \mathrm{~h}$. Aerobic growth of C. coli OR12 Aer was noted at $37^{\circ} \mathrm{C}$ but not at $42^{\circ} \mathrm{C}$. Microaerobic passage did not affect the ability of the C. coli OR12 Aer to grow aerobically, indicating that the aerotolerant phenotype is stable and likely due to genetic changes rather than temporary physiological adaptation. No difference in swarming motility was observed between the wild type and aerotolerant C. coli OR12. Further evidence of motility was provided by a membrane filtration assay, where growth occurred on BA following inoculation through a $0.45 \mu \mathrm{m}$ nitrocellulose filter. Impaired motility has been reported to significantly reduce the ability of campylobacters to pass through cellulose membranes (Speegle et al., 2009). This suggests that the aerobic growth can be initiated by motile cells and is not solely facilitated by a protective layer of non-viable cells, shielding viable cells from atmospheric oxygen. Aerotolerant C. coli OR12 demonstrated superior resistance to $\mathrm{H}_{2} \mathrm{O}_{2}$ than either wild type C. coli OR12 or C. coli RM2228. However, C. coli OR12 Aer pre-cultured under microaerobic conditions was more sensitive to $\mathrm{H}_{2} \mathrm{O}_{2}$ than an inoculum from an aerobic culture. Aerobic growth may therefore induce factors which render the cells less sensitive to peroxide stress.

Transmission electron microscopy was performed to determine whether the aerotolerant adaptation was accompanied by any changes in cell morphology, for example cell filamentation was noted by Ghaffar et al. (2015) upon entry to stationary phase in broth cultures. No differences in cell morphology were apparent between the wild type and aerotolerant C. coli OR12, with spiral, filamentous and coccid cells present within both populations.

Pulsed-field gel electrophoresis of four C. coli OR12 Aer isolates, which had been aerobically passaged in parallel, showed no difference to the parent strain. As well as confirming that the isolates were all $C$. coli OR12 and had not been contaminated, this suggested that the aerotolerance was not associated with any major recombination events, such as the genomic rearrangements observed in C. jejuni HPC5 in response to phage predation reported by Scott et al. (2007). The changes associated with aerotolerance were therefore considered more likely to be due to point mutations, and thus, whole genome sequencing was performed to investigate this. 


\section{Previous Reports of Aerotolerance}

Several authors have reported aerotolerance of $C$. jejuni and C. coli, however, the methods adopted to assess this are variable. For example, Jones et al. (1993) report aerobic growth of C. jejuni strains on blood agar after 2-3 days of aerobic incubation, but used an initial acclimation period of $18 \mathrm{~h}$ under microaerobic conditions. Similarly, Chynoweth et al. (1998) found 29 of 40 C. jejuni isolates from various sources could be adapted to grow aerobically on nutrient agar. All isolates were grown microaerobically before streaking on nutrient agar, and though some were initially slow to grow, they became adapted following aerobic subculture. Gaynor et al. (2004) reported the aerobic passage of C. jejuni NCTC 11168 on blood agar with antibiotic supplement at $37^{\circ} \mathrm{C}$, which resulted in significant attenuation of colonization in a day-old chick model after thirteen passages.

In contrast, Oh et al. (2015) examined aerotolerance of 70 C. jejuni isolates obtained from chicken meat samples with respect to survival of approximately $10^{9} \mathrm{CFU} / \mathrm{ml}$ in aerobic conditions at $42^{\circ} \mathrm{C}$ in MHB. Of these, 50 were considered aerotolerant based on survival for $12 \mathrm{~h}$ and 25 classified as hyperaerotolerant, with survival for $24 \mathrm{~h}$. The importance of alkyl hydroperoxide reductase was demonstrated by mutation of $a h p C$ leading to the loss of hyper aerotolerance (Oh et al., 2015). Purdy et al. (1999) studied the effect of $\operatorname{sodB}$ mutation on aerotolerance and in vivo colonization fitness of C. coli. Inactivation of sodB activity did not affect survival of cultures aerobically incubated at $25^{\circ} \mathrm{C}$ in $\mathrm{MHB}$, however, the colonization of day old chicks was reduced (Purdy et al., 1999). The aerotolerance test used by Purdy may not be equivalent to that described by Oh et al. (2015), as oxidative stress has been demonstrated to have a significantly greater effect at $42^{\circ} \mathrm{C}$ that at $25^{\circ} \mathrm{C}$ (Garénaux et al., 2008).

Kaakoush et al. (2007) examined the growth at several cell densities of four C. jejuni strains from aerobically and microaerobically incubated brain heart infusion broth. High initial bacterial density cultures grew when incubated either aerobically or microaerobically; however, cultures with less than $10^{4} \mathrm{CFU} / \mathrm{ml}$ only grew under microaerobic conditions. A combination of lower oxygen saturation in liquid media at $42^{\circ} \mathrm{C}$ and dense cultures that have a greater oxygen demand will lower the oxygen tension and allow growth. No growth occurred when C. coli OR12 Aer or WT were aerobically incubated at $37^{\circ} \mathrm{C}$ in MHB; indeed similar decline rates were observed for the C. coli OR12 derivatives and C. coli RM2228.

Rodrigues et al. (2015) recently reported the aerobic growth of C. jejuni strain $\mathrm{Bf}$ on Karmali agar. For this strain visible growth was reported within $24 \mathrm{~h}$ of aerobic incubation at $42^{\circ} \mathrm{C}$. Similar to C. coli OR12 Aer, growth in liquid medium could not be elicited, and a quantitative assay was used to measure growth on solid medium. Superior resistance to peroxide stress was also noted compared to C. jejuni NCTC 11168, which had failed to grow aerobically (Rodrigues et al., 2015). Growth on solid medium creates oxygen gradients, such that facultative anaerobes have been found to be more active on the bottom layer and aerobes more rapidly dividing on the top layer of colonies (Reyrolle and Letellier, 1979). Oxygen gradients in bacterial colonies and solid media have also been measured analytically, with the top $30 \mu \mathrm{m}$ of a gelatine medium being considered aerobic (Jeanson et al., 2015). Therefore, once initial growth of Campylobacter has commenced on a solid medium, bacteria that are not directly exposed to the outside atmosphere may reside in an atmosphere more similar to microaerobic conditions.

\section{Chicken Colonization}

Chicken colonization studies confirmed that wild type C. coli OR12 is a robust colonizing strain, with reproducible caecal counts between $10^{8}$ and $10^{9} \mathrm{CFU} / \mathrm{g}$. However, the aerotolerant strain colonized only $2 / 7$ birds at 3 days and $6 / 8$ birds by 7 days. Birds that were colonized had relatively high counts, with all $>7 \log _{10} \mathrm{CFU} / \mathrm{g}$. The binary nature of the outcomes suggests that bottlenecking events and stochastic processes are significant factors in the colonization process. They are also suggestive of adaptation occurring between days 3 and 7, where campylobacters, presumably present at levels below the limit of detection, have become acclimated to the intestinal or caecal environment and proliferate to achieve higher colonization levels. These adaptations may not be identical between birds, as suggested by the wider range of caecal counts compared with the original isolate. This experiment further validates the importance of individual caging of chickens during Campylobacter colonization assays as advocated by Conlan et al. (2011). Had the birds been group housed, any chickens in which the aerotolerant $C$. coli OR12 had colonized would likely have shed the modified strain in large numbers in feces, potentially rapidly colonizing the other birds in the group. The reduction in initial colonization fitness could have been missed under these circumstances. A reduction in the challenge dose could potentially allow further resolution between groups and may be of value in future studies. Aerotolerant C. coli OR12 recovered from chickens were still capable of aerobic growth demonstrating the trait is stable and compatible with chicken colonization, albeit potentially at a lower initial level. However, it is difficult to distinguish the lower levels of colonization from the overall effect of in vitro passage, which is known to reduce the colonization fitness (Stern et al., 1988). Nevertheless these findings correlate with the observations of Jones et al. (1993) in that aerotolerant C. jejuni recovered from mice retained aerotolerance. The evident adaptation between days 3 and 7 did not lead to any loss of aerotolerance. The ability of $C$. coli OR12 to adapt to aerobic conditions and re-adapt to chicken colonization may be a driver of diversity within the Campylobacter population, where the plastic genome allows rapid adaptation to new or stressful conditions. Given the capacity of campylobacters for interstrain recombination these changes may then be disseminated amongst cohabiting Campylobacter populations. The ability to withstand, and grow, in aerobic conditions could be of major importance not only in environmental persistence, allowing fomite transfer between farms, but could also allow prolonged survival on processed chicken carcasses and therefore be of public health importance. Survival of C. coli OR12 on chicken skin following refrigeration and freezing was examined by ElShibiny et al. (2009). Survival rates were comparable with the other C. jejuni and C. coli strains tested. Similar experiments with aerotolerant C. coli OR12 could provide valuable insight as to 
whether improved aerotolerance in vitro translates to superior survival on product, which would be of public health significance. This may be further considered in the context of the recently described aerobic C. jejuni strain Bf, which was found to be able to adhere to human epithelial cells and form biofilms under aerobic conditions (Bronnec et al., 2016).

This report describes true aerobic growth, without initial support from microaerobic incubation. With the exception of C. coli OR12, no growth was noted from any aerobically incubated Campylobacter spp. in this study. However, the equality of growth following aerobic or microaerobic incubation, which had been reported by Jones et al. (1993) was not achieved with C. coli OR12. Nevertheless the ability to undergo direct growth in atmospheric oxygen, a trait that was retained post either microaerobic growth or in vivo passage through chickens, encouraged us to examine the genetic basis for C. coli OR12 aerotolerance.

\section{Genomic Analysis}

Oxidative stress resistance has been proposed as a key feature of aerotolerance. Almost all the genes whose products have been implicated in oxidative stress resistance could be identified in C. coli OR12, none contained any mutations in the aerotolerant C. coli OR12. Orthologs of $\operatorname{rrp} A$ and $\operatorname{rrpB}$, encoding MarR-like transcription factors implicated in oxidative stress resistance, could not be identified in the genome sequences of $C$. coli OR12 or RM1875 or 15-537360 (Gundogdu et al., 2015). Bronnec et al. (2016) identified a complete type VI secretion system in C. jejuni Bf and suggested that it may contribute to the aerotolerant phenotype. The $C$. coli OR12 genome contains a complete T6SS, although $C$. coli RM2228, which did not demonstrate aerotolerance, also possesses a full T6SS (BleuminkPluym et al., 2013). This would suggest that possession of T6SS alone does not confer aerotolerance, although it could be involved when in combination with other features. Changes in 23 coding sequences were observed between the wild type and aerotolerant C. coli OR12 genomes. The functional significances of these are discussed in the following paragraphs.

\section{Phosphoglycerate Kinase}

Phosphoglycerate kinase (PGK) is a magnesium dependent kinase involved in glycolysis and gluconeogenesis. In many organisms it performs the first ATP- generating step in glycolysis, however, C. jejuni lacks a homolog of 6-phosphofructokinase and therefore cannot metabolize hexose sugars (Velayudhan and Kelly, 2002). Under these circumstances C. jejuni PGK is likely to be limited to the gluconeogenic pathway, converting 3-phosphoglycerate to 1,3-diphosphoglycerate (Figure 6).

The aerotolerant $C$. coli OR12 sequence has an insertion early in the $p g k$ gene that causes a frame shift and termination at 21 amino acids. No other copies of $p g k$ are present, so it is likely that no functional PGK protein is produced. This may prevent the gluconeogenic pathway from occurring in aerotolerant C. coli OR12, so sugar anabolism must be occurring via different pathways. The $C$ coli OR12 strain does possess orthologs to the serABC genes present in C. jejuni NCTC11168 (ATE51_01764; ATE51_03664; ATE51_03558, BLASTP identities of 94, 79,

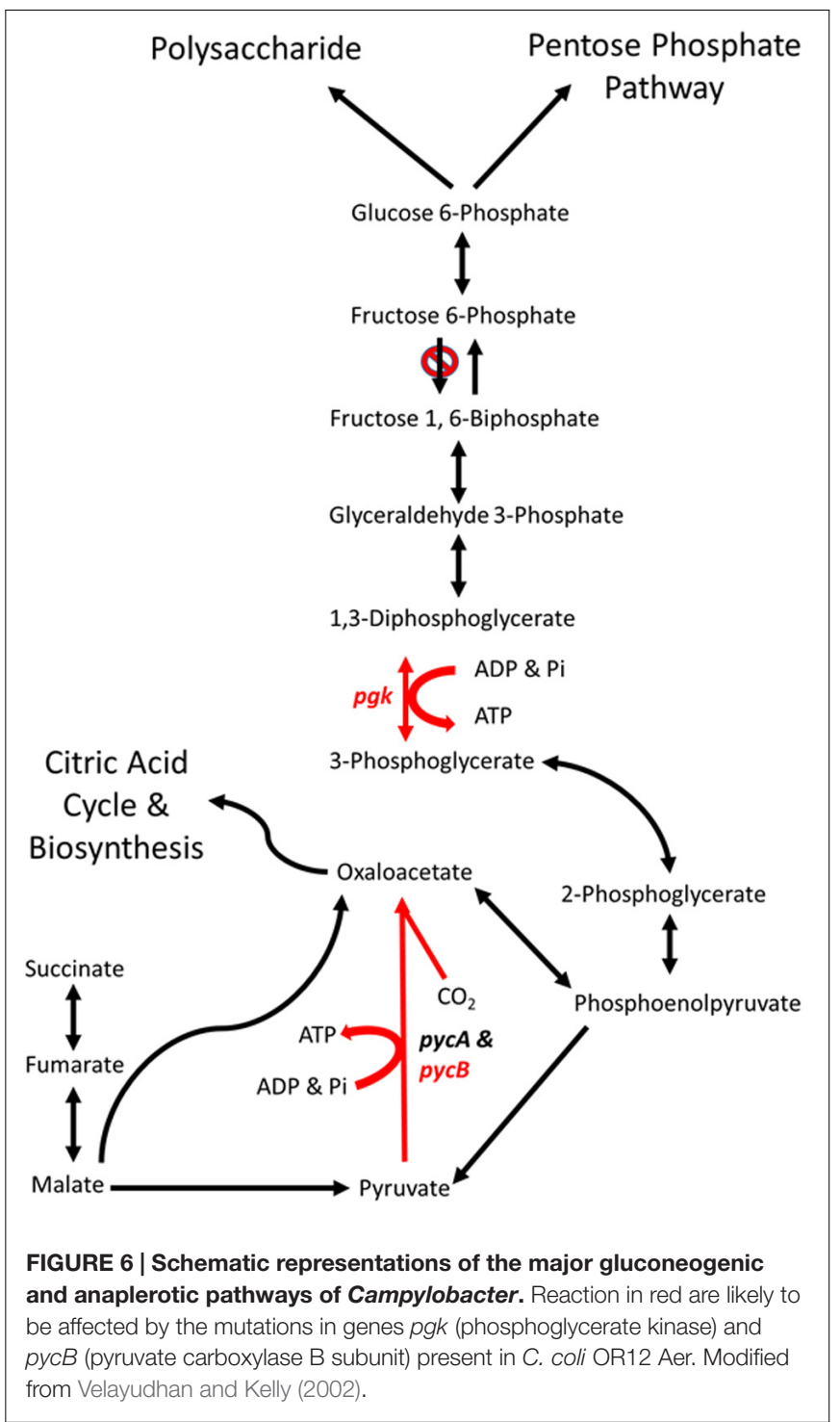

and $87 \%$, respectively). Therefore, serine biosynthesis from 3phosphoglycerate should be unaffected (Velayudhan et al., 2004). Circumvention of PGK and reinstatement of gluconeogenesis is theoretically possible via the formation of 2,3-bisphospho-Dglycerate, which would require the enzyme bisphosphoglycerate mutase. A putative phosphoglycerate/bisphosphoglycerate mutase has been annotated for C. jejuni NCTC11168, for which an ortholog is present in C. coli OR12 (ATE51_01624). The accumulation of 3-phosphoglycerate in the absence of PGK could drive this alternative reaction series. Aerobic growth of $C$. coli OR12 on BA plates prompted this study, and it is worth noting that erythrocytes are estimated to contain 4 to $5 \mathrm{mM}$ 2,3-bisphospho-D-glycerate formed by the LueberingRapoport glycolytic shunt, which is used to promote the release of oxygen from deoxygenated hemoglobin at times of need by binding the T-state as an allosteric effector (Benesch and Benesch, 1967). The availability of 2,3-bisphospho-Dglycerate, albeit at declining concentrations, could be utilized by 
campylobacters to reduce or remove the requirement of PGK in gluconeogenesis and increase the affinity of any remaining hemoglobin to bind oxygen. However, the adaptation present in the aerotolerant $C$. coli OR 12 also enables aerobic growth on CCDA. Modifications to central metabolism are emerging amongst campylobacters, for example it was recently reported that $1.7 \%$ of thermophilic campylobacters have the potential to metabolize glucose via the Entner-Doudoroff pathway (Vegge et al., 2016). This pathway also permits the metabolism of L-fucose, which has been reported to provide a competitive advantage to $C$. jejuni in a piglet colonization model (Stahl et al., 2011).

\section{Biotin Attachment Protein}

The cfiA gene in the wild-type $C$. coli OR12 sequence encodes a putative biotin attachment protein. The protein sequence shares $85 \%$ identity with $100 \%$ coverage of the pyruvate carboxylase B subunit of C. jejuni NCTC 11168. Therefore, this protein is likely to be the ortholog of $\mathrm{PycB}$, encoded by the gene cj0933c in C. jejuni NCTC 11168. Pyruvate carboxylase in C. jejuni is composed of two subunits, PycA and PycB (Velayudhan and Kelly, 2002). These correspond with the biotin carboxylase and biotin carboxyl carrier subunits of a two-subunit type of carboxylase (Goss et al., 1981). The complete enzyme contains biotin and catalyzes the ATP-dependent carboxylation of pyruvate, yielding oxaloacetate. Velayudhan and Kelly (2002) performed mutation studies of $p y c B$ and $p y c A$, and they noted that mutation of $p y c B$ resulted in significantly reduced growth in $\mathrm{BHI}$ broth, and the inability to use lactate, pyruvate or malate as carbon sources.

The C. coli OR12 Aer sequence contains a $\mathrm{T}$ insertion at nucleotide position 837,990 compared to the wild-type sequence. This would result in a frame shift and premature termination at 419 amino acids. This is likely to affect the activity of the enzyme as it is missing almost one third of its amino acids, including part of the conserved carboxylase domain as well as the entirety of the putative biotinylation and carboxylase interaction sites. This is surprising as the aerotolerant C. coli OR12 has a growth rate very similar to the wild type when grown microaerobically in NB2. Colonies on solid medium from microaerobically incubated aerotolerant C. coli OR12 were slightly smaller than the wild type, but this is not similar to the $p y c B$ mutant which had a threefold reduction in growth compared to the wild type (Velayudhan and Kelly, 2002). Comparisons of carbon metabolism in C. jejuni and C. coli indicate these species share a core set of carbon sources including amino acids, citric acid cycle intermediates and carboxylic acids (Wagley et al., 2014). However, exceptions were noted such as the inability of $C$. coli strains to metabolize D-malic acid and the inability of $C$. jejuni strains to metabolize propionic acid.

\section{PseD/Motility Accessory Factor}

Post-translational glycosylation of flagellar proteins is important for Campylobacter virulence. For example, mutation of pseA in C. jejuni 81-176 resulted in deficiency of flagellar glycosylation with pseudaminic acid and reduced virulence in a ferret model
(Guerry et al., 2006). The function of the gene pseD, cj01333 in C. jejuni NCTC 11168, which is a member of the motility accessory family, was examined in the context of flagellar glycosylation in C. jejuni 81-167 by McNally et al. (2006). Mutation of pseD did not affect cell motility or flagellin glycosylation with pseduaminic acid. However, mutation did prevent glycosylation with the pseudaminic acid acetamidino derivative PseAm, though it did not prevent cellular production of PseAm. Changes within a homopoylmeric $\mathrm{C}$ tract of $p s e D$ in aerotolerant $C$. coli OR12 effectively reinstates a longer reading frame to fuse ATE51_00982 and ATE51_00984 to create a 648 amino acid predicted product that exhibits $99 \%$ coverage with the C. jejuni NCTC 11168 PseD.

\section{Filamentous Haemagglutinin}

The gene $h x u A$ encodes a protein which shares high sequence identity with other $C$. coli proteins annotated as filamentous haemagglutinin. The sequence also contains a region with high similarity to several conserved haemagglutination activity domains. Filamentous haemagglutinin proteins are involved in virulence of several bacterial pathogens, including Bordetella pertussis and Avibacterium (formerly Haemophilus) paragallinarum (Hobb et al., 2002; Inatsuka et al., 2005). The genome of Arcobacter butzleri ATCC 49616 contains the gene $h c p A$ which encodes an iron activated filamentous hemagglutinin (Douidah et al., 2012). BLASTP comparison of this protein with $C$. coli OR12 HxuA revealed no significant similarity, nor did comparison with the C. concisus HcpA, the HagA protein of A. paragallinarum, or the Bordetella proteins FhaA and FhaB. C. fetus subsp. fetus 82-40 encodes a putative filamentous haemagglutinin of the HcpA family. It may be coincidental that this species is associated with bacteraemic infections, however, it is unknown whether this potential virulence factor is functional in vivo (Miller, 2008). The protein may have a possible role in iron acquisition or metabolism, which is known to be involved in the oxidative stress response (Atack and Kelly, 2009). The function of the putative filamentous haemagglutinin encoded by $h x u A$ in C. coli OR12 remains obscure but the reading frame is notable in that it is phase variable, being intact in $96 \%$ of the wild-type sequence reads but appears disrupted in aerotolerant C. coli OR12.

\section{Carbonic Anhydrases}

Carbonic anhydrase enzymes catalyze the reversible hydration of $\mathrm{CO}_{2}$ to bicarbonate and can be divided into at least six different phylogenetic groups, with varying expressions across kingdoms (Bury-Moné et al., 2008; Al-Haideri et al., 2015). These groups have similar function but differing structures, which are believed to have arisen by convergent evolution (Smith and Ferry, 2000). Strains of C. jejuni are reported to encode a $\beta$ and a $\gamma$ carbonic anhydrase, the former class are oligomeric, containing between 2 and 8 monomers and the latter are homotrimers (Smith and Ferry, 2000). However, the putative carbonic anhydrase annotated genes affected in the aerotolerant C. coli OR12 are not similar to those that have been investigated in campylobacters thus far. Whether these represent 
a different class of carbonic anhydrases not yet characterized in Campylobacter, or encode a hitherto unknown function requires further investigation.

Eleven other genes contained coding mutations, all of which involved single nucleotide substitutions or changes of up to three amino acids at the C-terminus. None of these involved genes which could be expected to influence aerotolerance, such as those encoding oxidative stress resistance proteins or their regulatory genes. Further investigation of these changes would include protein modeling software to determine whether the changes induce significant structural alterations or cause relocation of the protein.

\section{CONCLUSION}

The C. coli strain OR12, which is a robust colonizer of chickens, was found to be capable of aerobic growth on blood agar. This aerotolerance became further evident after serial passage and was associated with increased peroxide stress resistance but did not influence cellular morphology or motility. Colonization of chickens by aerotolerant $C$. coli OR12 was lower than the wildtype strain at 3 days after challenge but not by 7 days, suggesting adaptation was occurring. Whole genome sequencing did not reveal any changes in genes known to be related to oxidative stress. Instead, significant mutations were present within genes encoding phosphoglycerate kinase and pyruvate carboxylase, suggesting alterations to cellular metabolism.

\section{REFERENCES}

Al-Haideri, H., White, M. A., and Kelly, D. J. (2015). Major contribution of the type II beta carbonic anhydrase CanB (Cj0237) to the capnophilic growth phenotype of Campylobacter jejuni. Environ. Microbiol. 18, 721-735. doi: 10.1111/14622920.13092

Atack, J. M., Harvey, P., Jones, M. A., and Kelly, D. J. (2008). The Campylobacter jejuni thiol peroxidases Tpx and Bcp both contribute to aerotolerance and peroxide-mediated stress resistance but have distinct substrate specificities. J. Bacteriol. 190, 5279-5290. doi: 10.1128/JB.00100-08

Atack, J. M., and Kelly, D. J. (2008). Contribution of the stereospecific methionine sulphoxide reductases MsrA and MsrB to oxidative and nitrosative stress resistance in the food-borne pathogen Campylobacter jejuni. Microbiology 154, 2219-2230. doi: 10.1099/mic.0.2008/019711-0

Atack, J. M., and Kelly, D. J. (2009). Oxidative stress in Campylobacter jejuni: responses, resistance and regulation. Future Microbiol. 4, 677-690. doi: 10.2217/ fmb.09.44

Benesch, R., and Benesch, R. E. (1967). The effect of organic phosphates from the human erythrocyte on the allosteric properties of hemoglobin. Biochem. Biophys. Res. Commun. 26, 162-167. doi: 10.1016/0006-291X(67)90228-8

Blaser, M., and Engberg, J. (2008). "Clinical aspects of Campylobacter jejuni and Campylobacter coli infections," in Campylobacter, 3 Edn, eds I. Nachamkin, C. M. Szymanski, and M. J. Blaser (Washington DC: ASM Press), 99-121. doi: 10.1128/9781555815554.ch6

Bleumink-Pluym, N. M. C., van Alphen, L. B., Bouwman, L. I., Wösten, M. M. S. M., and van Putten, J. P. M. (2013). Identification of a functional type VI secretion system in Campylobacter jejuni conferring capsule polysaccharide sensitive cytotoxicity. PLoS Pathog. 9:e1003393. doi: 10.1371/journal.ppat. 1003393

Bolton, D. J. (2015). Campylobacter virulence and survival factors. Food Microbiol. 48, 99-108. doi: 10.1016/j.fm.2014.11.017

Bronnec, V., Turoňová, H., Bouju, A., Cruveiller, S., Rodrigues, R., Demnerova, K., et al. (2016). Adhesion, biofilm formation, and genomic features of

\section{AUTHOR CONTRIBUTIONS}

PO'K and IC designed and executed experiments, analyzed data, prepared and reviewed the manuscript.

\section{FUNDING}

This work was supported by the Biotechnology and Biological Sciences Research Council [grant number BB/I024585/1], UK. PO'K was supported by Biotechnology and Biological Sciences Research Council Doctoral Training Programme [grant number BB/J014508/1]. The chicken colonization experiment was funded by University of Nottingham Bio Services Unit strategic funding.

\section{ACKNOWLEDGMENT}

The assistance of Dr. Phillippa Connerton, Nicola Cummings, Geraldine Lafontaine, Antung S. Firleyanti, Lu Liang, and Philip Richards are gratefully acknowledged.

\section{SUPPLEMENTARY MATERIAL}

The Supplementary Material for this article can be found online at: http://journal.frontiersin.org/article/10.3389/fmicb. 2017.00513/full\#supplementary-material

Campylobacter jejuni Bf, an atypical strain able to grow under aerobic conditions. Front. Microbiol. 7:278. doi: 10.3389/fmicb.2016.01002

Brown, H. (2016). Spreadsheet for Sample Size Calculation, 2016 [Interactive Resource [Online]. Edinburgh: University of Edinburgh.

Bury-Moné, S., Mendz, G. L., Ball, G. E., Thibonnier, M., Stingl, K., Ecobichon, C., et al. (2008). Roles of $\alpha$ and $\beta$ carbonic anhydrases of Helicobacter pylori in the urease-dependent response to acidity and in colonization of the murine gastric mucosa. Infect. Immunol. 76, 497-509. doi: 10.1128/IAI. 00993-07

Chynoweth, R. W., Hudson, J. A., and Thom, K. (1998). Aerobic growth and survival of Campylobacter jejuni in food and stream water. Lett. Appl. Microbiol. 27, 341-344. doi: 10.1046/j.1472-765X.1998.00453.x

Conlan, A. J. K., Line, J. E., Hiett, K., Coward, C., Van Diemen, P. M., Stevens, M. P., et al. (2011). Transmission and dose-response experiments for social animals: a reappraisal of the colonization biology of Campylobacter jejuni in chickens. J. R. Soc. Interface 8, 1720-1735. doi: 10.1098/rsif.2011.0125

Douidah, L., de Zutter, L., Bare, J., De Vos, P., Vandamme, P., Vandenberg, O., et al. (2012). Occurrence of putative virulence genes in Arcobacter species isolated from humans and animals. J. Clin. Microbiol. 50, 735-741. doi: 10.1128/JCM. 05872-11

EFSA (2010). Analysis of the baseline survey on the prevalence of Campylobacter in broiler batches and of Campylobacter and Salmonella on broiler carcasses in the EU, 2008. EFSA J. 8, 1503. doi: 10.2903/j.efsa.2010.1503

EFSA (2015). The European Union summary report on trends and sources of zoonoses, zoonotic agents and food-borne outbreaks in 2014. EFSA J. 13, 4329.

EFSA (2016). The European Union summary report on trends and sources of zoonoses, zoonotic agents and food-borne outbreaks in 2015. EFSA J. 14, 4634.

El-Shibiny, A. (2006). Integral Quality Control Studies of Campylobacter on Poultry and Poultry Meat: Prevalence and Survival of Campylobacter and Bacteriophage from Free-Range and Organc Broiler Chickens. Ph.D. thesis, University of Nottingham, Nottingham.

El-Shibiny, A., Connerton, P. L., and Connerton, I. F. (2005). Enumeration and diversity of campylobacters and bacteriophages isolated during the rearing 
cycles of free-range and organic chickens. Appl. Environ. Microbiol. 71, 1259-1266. doi: 10.1128/AEM.71.3.1259-1266.2005

El-Shibiny, A., Connerton, P. L., and Connerton, I. F. (2007). Campylobacter succession in broiler chickens. Vet. Microbiol. 125, 323-332. doi: 10.1016/j. vetmic.2007.05.023

El-Shibiny, A., Scott, A., Timms, A., Metawea, Y., Connerton, P., and Connerton, I. (2009). Application of a group II Campylobacter bacteriophage to reduce strains of Campylobacter jejuni and Campylobacter coli colonizing broiler chickens. J. Food Prot. 72, 733-740. doi: 10.4315/0362-028X-72.4.733

Flint, A., Stintzi, A., and Butcher, J. (2016a). Stress responses, adaptation, and virulence of bacterial pathogens during host gastrointestinal colonization. Microbiol. Spectr. 4:VMBF-0007. doi: 10.1128/microbiolspec.VMBF-00072015

Flint, A., Stintzi, A., and Saraiva, L. M. (2016b). Oxidative and nitrosative stress defences of Helicobacter and Campylobacter species that counteract mammalian immunity. FEMS Microbiol. Rev. 40, 938-960. doi: 10.1093/femsre/ fuw025

Flint, A., Sun, Y. Q., and Stintzi, A. (2012). Cj1386 is an ankyrin-containing protein involved in heme trafficking to catalase in Campylobacter jejuni. J. Bacteriol. 194, 334-345. doi: 10.1128/JB.05740-11

Fouts, D. E., Mongodin, E. F., Mandrell, R. E., Miller, W. G., Rasko, D. A., Ravel, J., et al. (2005). Major structural differences and novel potential virulence mechanisms from the genomes of multiple Campylobacter species. PLoS Biol. 3:e15. doi: 10.1371/journal.pbio.0030015

Garénaux, A., Jugiau, F., Rama, F., Jonge, R., Denis, M., Federighi, M., et al. (2008). Survival of Campylobacter jejuni strains from different origins under oxidative stress conditions: effect of temperature. Curr. Microbiol. 56, 293-297. doi: 10.1007/s00284-007-9082-8

Gaynor, E. C., Cawthraw, S., Manning, G., MacKichan, J. K., Falkow, S., and Newell, D. G. (2004). The genome-sequenced variant of Campylobacter jejuni NCTC 11168 and the original clonal clinical isolate differ markedly in colonization, gene expression, and virulence-associated phenotypes. J. Bacteriol. 186, 503-517. doi: 10.1128/JB.186.2.503-517.2004

Ghaffar, N. M., Connerton, P. L., and Connerton, I. F. (2015). Filamentation of Campylobacter in broth cultures. Front. Microbiol. 6:657. doi: 10.3389/fmicb. 2015.00657

Gillespie, I. A., O’Brien, S. J., Frost, J. A., Adak, G. K., Horby, P., Swan, A. V., et al. (2002). A case-case comparison of Campylobacter coli and Campylobacter jejuni infection: a tool for generating hypotheses. Emerg. Infect. Dis. 8, 937-942. doi: 10.3201/eid0809.010817

Goss, J. A., Cohen, N. D., and Utter, M. F. (1981). Characterization of the subunit structure of pyruvate carboxylase from Pseudomonas citronellolis. J. Biol. Chem. 256, 11819-11825.

Guerry, P., Ewing, C. P., Schirm, M., Lorenzo, M., Kelly, J., Pattarini, D., et al. (2006). Changes in flagellin glycosylation affect Campylobacter autoagglutination and virulence. Mol. Microbiol. 60, 299-311. doi: 10.1111/j. 1365-2958.2006.05100.x

Gundogdu, O., da Silva, D. T., Mohammad, B., Elmi, A., Mills, D. C., Wren, B. W., et al. (2015). The Campylobacter jejuni MarR-like transcriptional regulators RrpA and RrpB both influence bacterial responses to oxidative and aerobic stresses. Front. Microbiol. 6:710. doi: 10.3389/fmicb.2015.00724

Hermans, D., Van Deun, K., Martel, A., Van Immerseel, F., Messens, W., Heyndrickx, M., et al. (2011). Colonization factors of Campylobacter jejuni in the chicken gut. Vet. Res. 42:82. doi: 10.1186/1297-9716-42-82

Hobb, R. I., Tseng, H.-J., Downes, J. E., Terry, T. D., Blackall, P. J., Takagi, M., et al. (2002). Molecular analysis of a haemagglutinin of Haemophilus paragallinarum. Microbiology 148, 2171-2179. doi: 10.1099/00221287-148-72171

Hwang, S., Kim, M., Ryu, S., and Jeon, B. (2011). Regulation of oxidative stress response by CosR, an essential response regulator in Campylobacter jejuni. PLoS ONE 6:e22300. doi: 10.1371/journal.pone.0022300

Imlay, J. A. (2013). The molecular mechanisms and physiological consequences of oxidative stress: lessons from a model bacterium. Nat. Rev. Microbiol. 11, 443-454. doi: $10.1038 /$ nrmicro3032

Inatsuka, C. S., Julio, S. M., and Cotter, P. A. (2005). Bordetella filamentous hemagglutinin plays a critical role in immunomodulation, suggesting a mechanism for host specificity. Proc. Natl. Acad. Sci. U.S.A. 102, 18578-18583. doi: 10.1073/pnas.0507910102
Jacobs-Reitsma, W., Lyhs, U., and Wagenaar, J. (2008). “Campylobacter in the food supply," in Campylobacter, 3 Edn, eds I. Nachamkin, C. M. Szymanski, and M. J. Blaser (Washington DC: ASM Press), 626-644.

Jeanson, S., Floury, J., Gagnaire, V., Lortal, S., and Thierry, A. (2015). Bacterial colonies in solid media and foods: a review on their growth and interactions with the micro-environment. Front. Microbiol. 6:71. doi: 10.3389/fmicb.2015. 01284

John, A., Connerton, P. L., Cummings, N., and Connerton, I. F. (2011). Profound differences in the transcriptome of Campylobacter jejuni grown in two different, widely used, microaerobic atmospheres. Res. Microbiol. 162, 410-418. doi: 10. 1016/j.resmic.2011.02.004

Jones, D. M., Sutcliffe, E. M., and Rios, R. (1993). Campylobacter jejuni adapts to aerobic metabolism in the environment. J. Med. Microbiol. 38, 145-150. doi: 10.1099/00222615-38-2-145

Kaakoush, N. O., Miller, W. G., De Reuse, H., and Mendz, G. L. (2007). Oxygen requirement and tolerance of Campylobacter jejuni. Res. Microbiol. 158, 644-650. doi: 10.1016/j.resmic.2007.07.009

Loc Carrillo, C., Atterbury, R. J., El-Shibiny, A., Connerton, P. L., Dillon, E., Scott, A., et al. (2005). Bacteriophage therapy to reduce Campylobacter jejuni colonization of broiler chickens. Appl. Environ. Microbiol. 71, 6554-6563. doi: 10.1128/AEM.71.11.6554-6563.2005

McNally, D. J., Hui, J., Aubry, A. J., and Mui, K. (2006). Functional characterization of the flagellar glycosylation locus in Campylobacter jejuni 81-176 using a focused metabolomics approach. J. Biol. Chem. 281, 18489-18498. doi: 10.1074/ jbc.M603777200

Miles, A. A., Misra, S. S., and Irwin, J. O. (1938). The estimation of the bactericidal power of the blood. J. Hyg. (Lond) 38, 732-749. doi: 10.1017/ S002217240001158X

Miller, W. G. (2008). "Comparative genomics of Campylobacter species other than Campylobacter jejuni," in Campylobacter, 3 Edn, eds I. Nachamkin, C. M. Szymanski, and M. J. Blaser (Washington DC: ASM Press), 73-95. doi: 10.1128/ 9781555815554.ch5

Moreno, G. S., Griffiths, P. L., Connerton, I. F., and Park, R. W. (1993). Occurrence of Campylobacters in small domestic and laboratory animals. J. Appl. Bact. 76, 49-54. doi: 10.1111/j.1365-2672.1993.tb03406.x

O'Brien, S. J., Larose, T. L., Adak, G. K., Evans, M. R., and Tam, C. C. (2016). Modelling study to estimate the health burden of foodborne diseases: cases, general practice consultations and hospitalisations in the UK, 2009. BMJ Open 6:e011119. doi: 10.1136/bmjopen-2016-011119

Oh, E., McMullen, L., and Jeon, B. (2015). High prevalence of hyper-aerotolerant Campylobacter jejuni in retail poultry with potential implication in human infection. Front. Microbiol. 6:28. doi: 10.3389/fmicb.2015.01263

Palyada, K., Sun, Y.-Q., Flint, A., Butcher, J., Naikare, H., and Stintzi, A. (2009). Characterization of the oxidative stress stimulon and PerR regulon of Campylobacter jejuni. BMC Genomics 10:481. doi: 10.1186/1471-2164-10-481

Parkhill, J., Wren, B. W., Mungall, K., Ketley, J. M., Churcher, C., Basham, D., et al. (2000). The genome sequence of the food-borne pathogen Campylobacter jejuni reveals hypervariable sequences. Nature 403, 665-668. doi: 10.1038/35001088

PHE (2013). Public Health England: Campylobacter Cases: 2000 to 2012. Available: https://www.gov.uk/government/publications/campylobacter-cases2000-to-2012 [accessed September 12, 2016].

Purdy, D., Cawthraw, S., Dickinson, J. H., Newell, D. G., and Park, S. F. (1999). Generation of a superoxide dismutase (SOD)-deficient mutant of Campylobacter coli: evidence for the significance of SOD in Campylobacter survival and colonization. Appl. Environ. Microbiol. 65, 2540-2546.

Reyrolle, J., and Letellier, F. (1979). Autoradiographic study of the localization and evolution of growth zones in bacterial colonies. J. Gen. Microbiol. 111, 399-406. doi: 10.1099/00221287-111-2-399

Ribot, E. M., Fitzgerald, C., Kubota, K., Swaminathan, B., and Barrett, T. J. (2001). Rapid pulsed-field gel electrophoresis protocol for subtyping of Campylobacter jejuni. J. Clin. Microbiol. 39, 1889-1894. doi: 10.1128/JCM.39.5.1889-1894. 2001

Rodrigues, R. C., Pocheron, A.-L., Hernould, M., Haddad, N., Tresse, O., and Cappelier, J.-M. (2015). Description of Campylobacter jejuni Bf, an atypical aero-tolerant strain. Gut Pathog. 7, 30. doi: 10.1186/s13099-015-0077-x

Rutherford, K., Parkhill, J., Crook, J., Horsnell, T., Rice, P., Rajandream, M. A., et al. (2000). Artemis: sequence visualization and annotation. Bioinformatics 16, 944-945. doi: 10.1093/bioinformatics/16.10.944 
Scott, A. E., Timms, A. R., Connerton, P. L., Loc Carrillo, C., Adzfa Radzum, K., and Connerton, I. F. (2007). Genome dynamics of Campylobacter jejuni in response to bacteriophage predation. PLoS Pathog. 3:e119. doi: 10.1371/journal. ppat.0030119

Sheppard, S. K., Dallas, J. F., Strachan, N. J. C., MacRae, M., McCarthy, N. D., Wilson, D. J., et al. (2009). Campylobacter genotyping to determine the source of human infection. Clin. Infect. Dis. 48, 1072-1078. doi: 10.1086/ 597402

Smith, K. S., and Ferry, J. G. (2000). Prokaryotic carbonic anhydrases. FEMS Microbiol. Rev. 24, 335-366. doi: 10.1111/j.1574-6976.2000.tb00546.x

Speegle, L., Miller, M. E., Backert, S., and Oyarzabal, O. A. (2009). Use of cellulose filters to isolate Campylobacter spp. from naturally contaminated retail broiler meat. J. Food Prot. 72, 2592-2596. doi: 10.4315/0362-028X-72.12.2592

Stahl, M., Friis, L. M., Nothaft, H., Liu, X., Li, J., Szymanski, C. M., et al. (2011). L-fucose utilization provides Campylobacter jejuni with a competitive advantage. Proc. Natl Acad. Sci. U.S.A. 108, 7194-7199. doi: 10.1073/pnas. 1014125108

Stern, N., Bailey, J., Blankenship, L., and Cox, N. (1988). Colonization characteristics of Campylobacter jejuni in chick ceca. Avian. Dis. 32, 330-334. doi: 10.2307/1590822

Takamiya, M., Ozen, A., Rasmussen, M., Alter, T., Gilbert, T., Ussery, D. W., et al. (2011). Genome sequences of two stress-tolerant Campylobacter jejuni poultry strains, 305 and DFVF1099. J. Bacteriol. 193, 5546-5547. doi: 10.1128/JB. 05753-11

Tam, C. C., and O’Brien, S. J. (2016). Economic cost of Campylobacter, Norovirus and Rotavirus disease in the United Kingdom. PLoS ONE 11:e0138526. doi: 10.1371/journal.pone.0138526

Theoret, J. R., Cooper, K. K., Zekarias, B., Roland, K. L., Law, B. F., Curtiss, R., et al. (2012). The Campylobacter jejuni Dps homologue is important for in vitro biofilm formation and cecal colonization of poultry and may serve as a protective antigen for vaccination. Clin. Vaccine Immunol. 19, 1426-1431. doi: 10.1128/CVI.00151-12

van Gerwe, T., Bouma, A., Wagenaar, J. A., Jacobs-Reitsma, W. F., and Stegeman, A. (2010). Comparison of Campylobacter levels in crops and ceca of broilers at slaughter. Avian. Dis. 54, 1072-1074. doi: 10.1637/9113-101809ResNote.1

Vegge, C. S., Jansen van Rensburg, M. J., Rasmussen, J. J., Maiden, M. C. J., Johnsen, L. G., Danielsen, M., et al. (2016). Glucose metabolism via the entner-doudoroff pathway in Campylobacter: a rare trait that enhances survival and promotes biofilm formation in some isolates. Front. Microbiol. 7:1877. doi: 10.3389/fmicb. 2016.01877

Velayudhan, J., Jones, M. A., and Barrow, P. A. (2004). L-serine catabolism via an oxygen-labile L-serine dehydratase is essential for colonization of the avian gut by Campylobacter jejuni. Infect. Immun. 72, 260-268. doi: 10.1128/IAI.72.1. 260-268.2004

Velayudhan, J., and Kelly, D. J. (2002). Analysis of gluconeogenic and anaplerotic enzymes in Campylobacter jejuni: an essential role for phosphoenolpyruvate carboxykinase. Microbiology 148, 685-694. doi: 10.1099/00221287-148-3-685

Wagley, S., Newcombe, J., Laing, E., Yusuf, E., Sambles, C. M., Studholme, D. J., et al. (2014). Differences in carbon source utilisation distinguish Campylobacter jejuni from Campylobacter coli. BMC Microbiol. 14:262. doi: 10.1186/s12866014-0262-y

WHO (2013). The Global View of Campylobacteriosis: Report of an Expert Consultation. Geneva: World Health Organization.

Wilson, D. J., Gabriel, E., Leatherbarrow, A. J., Cheesbrough, J., Gee, S., Bolton, E., et al. (2008). Tracing the source of Campylobacteriosis. PLoS Genet. 4:e1000203. doi: 10.1371/journal.pgen.1000203

Conflict of Interest Statement: The authors declare that the research was conducted in the absence of any commercial or financial relationships that could be construed as a potential conflict of interest.

Copyright (c) 2017 O'Kane and Connerton. This is an open-access article distributed under the terms of the Creative Commons Attribution License (CC BY). The use, distribution or reproduction in other forums is permitted, provided the original author(s) or licensor are credited and that the original publication in this journal is cited, in accordance with accepted academic practice. No use, distribution or reproduction is permitted which does not comply with these terms. 